\title{
Optimized EGFR blockade strategies in EGFR addicted gastroesophageal adenocarcinomas
}

Simona Corso $^{1,2 \S} *$, Filippo Pietrantonio ${ }^{3,4 \S}$, Maria Apicella ${ }^{2 \S}$, Cristina Migliore ${ }^{1,2 \S}$, Daniela Conticelli ${ }^{1,2}$, Annalisa Petrelli ${ }^{2}$, Laura D’Errico ${ }^{1,2}$ Stefania Durando $^{2}$, Daniel Moya-Rull ${ }^{2}$, Sara E. Bellomo $^{2}$, Stefano Ughetto ${ }^{1,2}$, Maurizio Degiuli ${ }^{5}$, Rossella Reddavid ${ }^{5}$, Uberto Fumagalli ${ }^{6 \ddagger}$, Stefano De Pascale ${ }^{6 \ddagger}$, Giovanni Sgroi ${ }^{7}$, Emanuele Rausa ${ }^{7}$, Gian Luca Baiocchi ${ }^{8}$, Sarah Molfino ${ }^{8}$, Giovanni De Manzoni ${ }^{9}$, Maria Bencivenga ${ }^{9}$, Salvatore Siena ${ }^{4,10}$, Andrea Sartore-Bianchi ${ }^{4,10}$, Federica Morano $^{3}$, Salvatore Corallo $^{3}$, Michele Prisciandaro $^{3}$, Maria Di Bartolomeo ${ }^{3}$, Annunziata Gloghini ${ }^{11}$, Silvia Marsoni ${ }^{2} \ddagger$, Antonino Sottile ${ }^{2}$, Anna Sapino ${ }^{2,12}$, Caterina Marchio,2,12, Asa Dahle-Smith ${ }^{13}$, Zosia Miedzybrodzka ${ }^{14}$, Jessica Lee ${ }^{15}$, Siraj M. Ali ${ }^{15}$, Jeffrey S. Ross ${ }^{15,16}$, Brian Alexander ${ }^{15}$, Vincent A. Miller ${ }^{15}$, Russell Petty ${ }^{17}$, Alexa B. Schrock ${ }^{15}$ and Silvia Giordano ${ }^{1,2} *$.

${ }^{1}$ Department of Oncology, University of Torino, Candiolo, Italy; ${ }^{2}$ Candiolo Cancer Institute, FPOIRCCS, Candiolo, Italy; ${ }^{3}$ Medical Oncology Department, Fondazione IRCCS Istituto Nazionale dei Tumori, Milan, Italy; ${ }^{4}$ Department of Oncology and Hemato-oncology, University of Milan, Milan, Italy; ${ }^{5}$ Department of Oncology, University of Torino, Orbassano, Italy; ${ }^{6}$ Chirurgia Generale 2, Spedali Civili, Brescia, Italy; ${ }^{7}$ Surgical Oncology Unit, Surgical Science Department, ASST Bergamo Ovest, Treviglio (BG), Italy; ${ }^{8}$ Department of Clinical and Experimental Sciences, Surgical Clinic, University of Brescia, Brescia, Italy; ${ }^{9}$ Department of Surgical Sciences, Dentistry, Gynecology and Pediatrics, Section of Surgery, University of Verona, Italy; ${ }^{10}$ Niguarda Cancer Center, Grande Ospedale Metropolitano Niguarda, Milan, Italy; ${ }^{11}$ Pathology and Laboratory Medicine Department, Fondazione IRCCS Istituto Nazionale dei Tumori, Milan, Italy; ${ }^{12}$ Department of Medical Sciences, University of Torino, Italy; ${ }^{13}$ Tayside Cancer Centre, Ninewells Hospital, Dundee, Scotland, UK; ${ }^{14}$ University of Aberdeen, Aberdeen, UK; ${ }^{15}$ Foundation Medicine, 
Inc., Cambridge, USA; ${ }^{16}$ Dept of Pathology, Upstate Medical University, Syracuse, NY, USA;

${ }^{17}$ Division of Cancer Research, School of Medicine, University of Dundee, Dundee, Scotland, UK.

$\S$ These authors equally contributed to the work

+ Current Affiliation: Chirurgia dell'Apparato Digerente - European Institute of Oncology IRCCS Milan, Italy.

Running Title: EGFR Targeting in gastroesophageal adenocarcinoma

\section{Correspondence}

*Silvia Giordano and Simona Corso

Department of Oncology, University of Torino; Candiolo Cancer Institute, FPO-IRCCS, Strada

Provinciale 142, Candiolo, 10060 (Torino), Italy. Phone: +39 011 9933233/8; Fax: +39 011

9933225; e-mail: silvia.giordano@unito.it; simona.corso@unito.it

\section{Disclosures}

FP received honoraria from Amgen, Roche, Lilly, Bayer, Servier, Merck-Serono, Sanofi. Research grants from BMS. CM has received personal consultancy fees from Bayer, Roche, MSD, Daiichi Sankyo. AS-B. Participated in advisory boards for Amgen, Bayer, Samsung Bioepis and Sanofi. JL, SMA, JSR, BA, VAM and ABS are employees of Foundation Medicine, a wholly-owned subsidiary of Roche, with stock ownership in Roche. The remaining authors declare that they have no conflict of interest. All institutional and national guidelines for the care and use of laboratory animals were followed. 


\section{Abbreviations:}

COG: Cancer Oesophagus Gefitinib (Gefitinib for oesophageal cancer progressing after chemotherapy (COG): a phase 3, multicentre, double-blind, placebo-controlled randomised trial) FMI: Foundation Medicine, Inc. (Cambridge, Massachusetts, USA)

GEA: Gastric and gastroesophageal adenocarcinoma

INT: Istituto Nazionale Tumori (Milan, Italy)

IRCC: Istituto per la Ricerca e la Cura del Cancro (Candiolo, Torino, Italy)

PDX: Patient-derived xenograft 


\section{TRANSLATIONAL RELEVANCE}

Prior clinical trials performed in unselected gastroesophageal adenocarcinoma patients failed to show survival improvement upon treatment with anti-EGFR therapies. We report the clinical activity of EGFR monoclonal antibodies in patients bearing high level (>8 copies) of EGFR gene amplification and show that in Patient-derived xenografts the combination of an EGFR mAb and a tyrosine kinase inhibitor is significantly more effective and long lasting than mAb monotherapy. We also identify mTOR pathway activation as a novel mechanism of resistance to EGFR targeted therapy and show that it can be overcome by the combination of EGFR/mTOR inhibitors. These findings recognize EGFR as an actionable target in a small but significant subgroup of patients bearing EGFR amplification and suggest the combination of a EGFR mAb and a TKI as the most effective treatment. 


\section{ABSTRACT}

Purpose: Gastric and gastroesophageal adenocarcinomas (GEA) represent the third leading cause of cancer mortality worldwide. Despite significant therapeutic improvement, the outcome of patients with advanced GEA is poor. Randomized clinical trials failed to show a significant survival benefit in molecularly unselected patients with advanced GEA treated with anti-EGFR agents.

Experimental Design: We performed analyses on 4 cohorts: IRCC (570 patients), FMI (9397 patients), COG (214 patients) and INT (206 patients). Preclinical trials were conducted in patientderived xenografts (PDXs).

Results: The analysis of different GEA patient cohorts suggests that EGFR amplification drives aggressive behaviour and poor prognosis. We also observed that EGFR inhibitors are active in patients with $E G F R$ copy number gain and that co-amplification of other receptor tyrosine kinases or KRAS is associated with worse response. Pre-clinical trials performed on EGFR-amplified GEA PDX models revealed that the combination of an EGFR monoclonal antibody and an EGFR tyrosine kinase inhibitor was more effective than each monotherapy and resulted in a deeper and durable response. In a highly EGFR amplified non-responding PDX, where resistance to EGFR drugs was due to inactivation of the TSC2 tumor suppressor, co-treatment with the mTOR inhibitor everolimus restored sensitivity to EGFR inhibition.

Conclusions: This study underscores EGFR as a potential therapeutic target in gastric cancer and identifies the combination of an EGFR TKI and a monoclonal antibody as an effective therapeutic approach. Finally, it recognizes mTOR pathway activation as a novel mechanism of primary resistance that can be overcome by the combination of EGFR and mTOR inhibitors.

Keywords: EGFR; targeted therapy; gastric and gastroesophageal cancer; drug resistance; mTOR 


\section{INTRODUCTION}

Gastric and gastroesophageal adenocarcinomas (GEA) represent the third leading cause of cancerrelated deaths worldwide. Despite the introduction of novel systemic treatment options, the outcome of patients with metastatic gastroesophageal adenocarcinoma (mGEA) is still extremely unsatisfactory, with median overall survival (OS) of less than 12 months in most clinical trials (1). While the identification of specific molecular subtypes has had profound implications for targeted strategies in other malignancies, the same progress has only been partially realized for patients with mGEA. Trastuzumab and ramucirumab (targeting HER2 and VEGFR2, respectively) are the only approved targeted agents in mGEA $(2,3)$, whereas the promising role of immune checkpoint inhibitors (ICIs) such as pembrolizumab and nivolumab still needs to be confirmed by randomized clinical trials (RCTs) performed in properly selected patient subgroups.

The molecular landscape of GEA has been extensively described and the two main molecular classifications $(4,5)$ identified a disease subtype characterized by chromosomal instability and amplification of receptor tyrosine kinases (RTKs). Epidermal growth factor receptor (EGFR) amplification has been reported in 3-5\% of GEAs $(4,6)$, while other genetic alterations (such as point mutations or translocations) are extremely uncommon. Several EGFR targeting drugs, comprising monoclonal antibodies (MoAbs) and tyrosine kinase inhibitors (TKIs), have been approved for the treatment of multiple tumor types, including $R A S$ wild-type metastatic colorectal cancer, head and neck squamous cell carcinoma and EGFR mutated advanced non-small cell lung cancer (7). Conversely, three phase III Randomized Clinical trials evaluating the addition of cetuximab, panitumumab or gefitinib to the standard of care in molecularly unselected patients with advanced gastric or esophageal adenocarcinomas reported negative results (8-10). On the other hand, intriguingly, experimental data obtained in GEA preclinical models showed a positive correlation between cetuximab response and high EGFR expression/amplification (11). Consistent with these preclinical findings, the association between EGFR copy number gain (CNG) and better 
OS has been shown by a phase II trial of cetuximab plus FOLFOX chemotherapy in patients with mGEA (12). In addition, a pre-specified subgroup analysis of the COG trial showed that patients with esophageal and gastroesophageal junction carcinomas bearing EGFR CNG derived a significant progression-free survival (PFS), OS and Health-Related Quality of Life (HRQoL) benefit from gefitinib compared to placebo, thereby providing the proof-of-concept for EGFR CNG as a predictive biomarker of efficacy of EGFR targeted agents (13).

Here we aimed to investigate the efficacy of several EGFR inhibition strategies in preclinical models of EGFR amplified GEAs, to describe the clinical and molecular features of patients with EGFR amplified tumors and their responsiveness to EGFR inhibition, and to extensively investigate common and potentially novel genomic mechanisms of resistance, with the ultimate goal to optimize EGFR targeted combinations for the development of future clinical trials. 


\section{MATERIALS AND METHODS}

\section{Patients}

$\underline{\text { IRCC: }}$ Tumor samples (from gastric and gastroesophageal junction adenocarcinomas) and matched normal samples were obtained from patients undergoing surgery in 15 Italian Hospitals: Candiolo Cancer institute- FPO, IRCCS (Torino); Ordine Mauriziano Hospital; San Giovanni Battista Hospital (Torino); San Luigi Gonzaga Hospital, Orbassano (Torino); Humanitas-IRCCS, Rozzano, Milano; San Raffaele Hospital (Milano); Treviglio-Caravaggio Hospital (Bergamo); Brescia Hospital; Borgo-Trento Hospital (Verona); Santa Maria delle Scotte Hospital (Siena); Forli' Hospital; Fondazione Macchi Hospital (Varese); Pisa Hospital; Fondazione IRCCS Istituto Nazionale dei Tumori (Milano); Ospedale Niguarda Ca' Granda (Milano). All patients provided written informed consent; samples were collected and the study was conducted under the approval of the Review Boards of all the Institutions. The study was done in accordance with the principles of the Declaration of Helsinki, the International Conference on Harmonization and Good Clinical Practice guidelines and GDPR (General Data Protection Regulation). Clinical and pathologic data were entered and maintained in our prospective database. All the samples have been anonymized before being shipped to Candiolo. No reference to the patients can be inferred from the histological and molecular characterization presented in the work.

FMI: Tumor samples from patients with GEA were submitted during routine clinical care for CGP. Approval for this study, including a waiver of informed consent and a Health Insurance Portability and Accountability Act waiver of authorization, was obtained from the Western Institutional Review Board (protocol no. 20152817).

\section{Cell lines and drugs}

293 T cells were obtained from ATCC, OE21 from Sigma Aldrich (Saint Luis, MI, USA). The genetic identity of the cell lines was confirmed by short tandem repeat profiling (Cell ID, Promega, 
Madison, WI, USA). Erlotinib and everolimus were purchased from Carbosynth (UK). Cetuximab and lapatinib were provided by the Hospital Pharmacy.

\section{Primary cell cultures and organoids}

GEA primary cells were derived from PDXs as described in(14), while GEA primary organoids were obtained as described in (15). The genetic identity of the in vitro-derived material with the original tumor has been verified by short tandem repeat profiling (Cell ID, Promega). GTR0078 cells were used for the in vitro experiments soon after tumor dissociation, as they do not permanently grow in culture.

\section{Western blot analysis and immunoprecipitation}

Cells/organoids were treated with the indicated drugs: lapatinib or erlotinib $100 \mathrm{nM}$ for 2 hours; cetuximab $0.5 \mu \mathrm{g} / \mathrm{ml}$ for 16 hours. Whole-protein extracts were prepared using Laemmli buffer and quantified using the BCA Protein Assay kit (Pierce, Rockford, IL, USA). EGFR immunoprecipitation was performed with cetuximab on organoids (stimulated with EGF 100ng/ml for $15^{\prime}$, treated or not with erlotinib $100 \mathrm{nM}$ for $2 \mathrm{~h}$ ) previously washed out from matrigel with Cell Recovery Solution (\#354253, Corning, Glendale, AZ, USA) and lysed with EB (Triton 1\%, Tris$\mathrm{HCl}$ pH7.4 20 mM, EDTA pH8 5mM, Glycerol 10\%, NaCl 150mM). Primary antibodies: antiEGFR (1005:sc-03) and anti-Actin were from Santa Cruz Biotechnology (Inc., Dallas, Texas, USA), antibodies against phosphorylated EGFR (Tyr 845), ERK (Thr202/Tyr204), phosphorylated AKT (Ser473) (Clone D9E), total AKT, and ERK were from Cell Signaling (MA, USA). Antibody against phosphorylated EGFR (Tyr1068) (ab5644) was from Abcam (Cambridge, UK). Antibody directed against aa 1172-1186 of human EGFR was described in (16). Antibody anti-EGFR extracellular epitope (111.6 antibody) was from Thermo Fisher Scientific (MA, USA). Secondary antibodies were from Amersham. Detection was performed with ECL system (Amersham, UK).

\section{Transfection and transduction procedures}

OE21 cells were transfected with siRNAs using Lipofectamine2000 (Thermo Fisher Scientific). Transfection reagents plus siRNAs at final concentration of $20 \mathrm{nM}$ were used following standard 
protocols. Seventy two hours after transfection, cells were lysed and WB performed. TSC2 silencing was achieved using SMARTpool ON-TARGETplus siRNA (Dharmacon, Lafayette, CO, USA).

Lentiviruses were produced as described in (17). OE21 cells were transduced with a pool of lentiviral particles containing of four TSC2 silencing shRNAs (Sigma, \#40179, \#40178, \#40454, \#40455). Cells were selected with puromicin, checked for TSC2 silencing and subcutaneously injected in NOD/SCID mice $\left(5^{*} 10^{\wedge}\right.$ cells/mouse) in SF medium: Matrigel (Corning) $1: 1$.

\section{Analyte extraction}

Genomic DNA was isolated using the Blood \& Cell Culture DNA Midi Kit (Qiagen, Germany). DNA concentrations were quantified using the Qubit Fluorometer (Thermo Fisher Scientific).

\section{CNV Evaluation by qReal Time PCR}

Quantitative PCR experiments for estimation of EGFR, MET, FGFR2 and KRAS copy number variations were performed in triplicates using 2 ng total gDNA as a template, with the following Human TaqMan Copy Number Assays: for HER2 assay ID Hs02876245_cn, for EGFR assay ID Hs04942325_cn, for MET assay ID: Hs04993403_cn, for FGFR2 assay ID Hs01472955_cn, for KRAS assay ID Hs06936191 and the TaqMan Copy Number Reference Assay RNase P 4316831 and GREB1 Hs01738470_cn (Applied Biosystems, Foster City, CA, USA). PCR runs were performed with ABI Prism 7900HT (Applied Biosystems).

\section{AMNESIA panel}

In a case-control study setting, we identified a panel of gene alterations (including EGFR/MET/KRAS/PI3K/PTEN mutations and EGFR/MET/KRAS amplifications) able to predict primary resistance to trastuzumab therapy in HER2-positive metastatic gastric cancer patients (18). We applied the same panel of gene alterations (substituting EGFR mutation/amplification with HER2 mutation/amplification) in the context of EGFR driven tumors.

\section{Phospho- Kinase Array}

Cells were treated with the indicated drugs: lapatinib or erlotinib $100 \mathrm{nM}$ for 2 hours; cetuximab $0.5 \mu \mathrm{g} / \mathrm{ml}$ for 16 hours. The analysis of the phosphorylation profiles of kinases was performed using 
the Human Phospho-Kinase Antibody Array (R\&D Systems, Minneapolis, MN, USA), according to manufacturer instructions. Signal quantification was performed using Image Lab 5.2.1 Software (BioRad, Hercules, CA, USA).

\section{PDX generation}

Gastric PDX generation was performed as described in (19). All animal procedures adhered to the 'Animal Research: Reporting of In Vivo Experiments' (ARRIVE) standards and were approved by the Ethical Commission of the Candiolo Cancer Institute (Candiolo, Torino, Italy), and by the Italian Ministry of Health.

\section{PDX xenotrials}

PDXs were passaged and expanded for $>2$ generations until production of a cohort of mice. Established and randomized tumors (average volume $250 \mathrm{~mm}^{3}$ ) were treated for the indicated days with the following regimens (either single agent or combination): vehicle (saline) per os; cetuximab $20 \mathrm{mg} / \mathrm{Kg}$, twice weekly ip; lapatinib $100 \mathrm{mg} / \mathrm{kg}$, daily, per os; erlotinib $50 \mathrm{mg} / \mathrm{kg}$, daily, per os; everolimus $6 \mathrm{mg} / \mathrm{kg}$, daily, per os. Tumor size was evaluated once-weekly by caliper measurements and approximate volume of the mass was calculated using the formula $4 / 3 \pi(D / 2)(d / 2)^{2}$, where $d$ is the minor tumor axis and $\mathrm{D}$ is the major tumor axis. The response in mice has been evaluated using RECIST 1.1-like criteria, i.e. progressive disease (PD): $\geq 35 \%$ increase from baseline; partial response $(\mathrm{PR}): \geq 50 \%$ reduction from baseline; stable disease $(\mathrm{SD})$ : intermediate variations from baseline (20). Statistical testing for pharmacological experiment was performed with GraphPAD PRISM Software 8.0, using Two-way ANOVA followed by Bonferroni multiple comparisons experiments. Statistical significance: $n s=$ not significant; $* \mathrm{p}<0,05 ; * * \mathrm{p}<0,01 ; * * * \mathrm{p}<0,001$.

\section{Genomic sequencing}

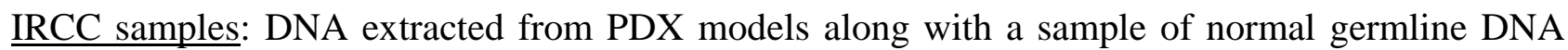
from each patient were utilized for next generation sequencing. Using standard methods, Illumina sequencing libraries were generated and subjected to hybrid capture with a focused targeted bait set 
of 243 genes selected based upon their alteration in prior studies of gastroesophageal cancer $(21,22)$.

FMI samples: Comprehensive genomic profiling (CGP) was performed in a Clinical Laboratory Improvement Amendments (CLIA)-certified, New York State and CAP (College of American Pathologists)-accredited laboratory (Foundation Medicine, Inc., Cambridge, MA). In brief, $\geq 50 \mathrm{ng}$ DNA was extracted from 40 microns of formalin-fixed, paraffin-embedded (FFPE) tissue blocks from 4,337 cases of gastric carcinoma. The samples were assayed by CGP using adaptor-ligation and hybrid capture was performed for all coding exons of from 180-395 cancer related genes plus select introns from 14-34 genes frequently rearranged in cancer. Sequencing of captured libraries was performed to a mean exon coverage depth of $>500 \mathrm{X}$, and resultant sequences were analyzed for genomic alterations including mutations (base substitutions, insertions and deletions), copy number alterations (focal amplifications and homozygous deletions), and select gene fusions or rearrangements, as previously described(23). EGFR amplification was defined as $E G F R$ copy $\geq 8$.

COG samples: RTK copy numbers were determined using Affymetrix OncoScan ${ }^{\circledR}$ CNV FFPE Assay following the manufacturer recommended protocol. DNA was extracted from histologically confirmed oesophageal and GEJ adenocarcinomas as described previously(13) and quantified using the Quant-iT ${ }^{\mathrm{TM}}$ PicoGreen ${ }^{\circledR}$ dsDNA Assay Kit (Life Technologies) following the manufacturer's recommended protocol, using 80ng for each case, normalised to a concentration $12 \mathrm{ng} / \mu \mathrm{L}$. Array fluorescence intensity data (CEL files), generated by Affymetrix ${ }^{\circledR}$ GeneChip ${ }^{\circledR}$ Command Console ${ }^{\circledR}$ (AGCC) Software version 4.0 were processed using OncoScan ${ }^{\circledR}$ Console software version 1.1.034 to produce OSCHP files and a set of QC metrics. Features were quantile normalised and genome wide allele specific copy number assessed using the Affymetrix TuScan algorithm to allow adjustment for both tumor ploidy and nonaberrant cell admixture(24). Genome wide copy number variation was assessed across all cases using AffymetrixNexus Express for OncoScan (version 3.1.). Significant copy number variation events across the genome were identified using a 'Significance Testing for Aberrant Copy number' (STAC) approach(25). 
INT samples: Formalin-fixed paraffin-embedded archival tumor tissue blocks obtained prior to any treatment were used for the purpose of this study. Next-generation sequencing was performed as in (26), to detect gene mutations, whereas EGFR, HER2 and MET status were determined by SISH analysis and KRAS GCN gain was assessed by PCR, as previously described in (18).

\section{Survival analysis}

OS was calculated from the date of enrollment (for the COG trial) or from the date of diagnosis of metastatic disease (for the INT dataset) until the date of death or last follow-up for alive patients. The OS curves for EGFR amplified vs non-amplified subgroups were calculated with the KaplanMeier method and compared with the log-rank test. Survival analysis for COG was undertaken using IBM SPPS statistics 22, further details see(10,13).

\section{In situ Hybridization and Immunohistochemistry}

EGFR gene status was assessed by bright-field dual-color SISH (Ventana Medical Systems). The Colorado scoring system was adopted to classify samples into ISH strata according to the frequency of cells with each EGFR gene copy number and referred to the chromosome 7 centromere. EGFR SISH-negative cases had no or low genomic gain for EGFR gene copy number (disomy, low trisomy, high trisomy, and low polysomy), whereas the distinction between high polysomy and gene amplification was defined by the presence of gene clusters only in EGFR-amplified cases. EGFR FISH in the COG cohort was performed and scored as described in (13).

IHC for EGFR was performed using the CONFIRM® anti-EGFR (5B7) rabbit monoclonal primary antibody (Ventana Medical Systems, Tucson, AZ) that recognizes the internal domain of EGFR and the monoclonal mouse anti human anti-EGFR (E30) antibody (Dako, Glostrup, Denmark) that recognizes an external domain of EGFR. IHC was carried out on an automated immunostainer (BenchMark Ultra; Ventana Medical Systems) using the Optiview DAB Detection Kit (Ventana Medical Systems). IHC for P-EGFR was performed using anti-P-EGFR Y1173 53AS from Cell Signaling (MA, USA).

\section{Transcriptome profiling}


RNA-seq libraries were prepared using the Illumina TruSeq Stranded Total RNA Library Prep Gold kit and sequenced generating 75 bp paired-end reads. PDX RNA-seq data were first deconvoluted for mouse contamination with Xenome (27) software (version 1.0.1). Non-host reads (those classified as "graft", "ambiguous" or "both") were then mapped to UCSC hg38 reference genome with HISAT2 (28) aligner with default parameters. Gene expression estimate was performed with HTSeq (29) in "intersection-nonempty" mode against GENCODE v33 annotation. 


\section{RESULTS}

\section{Prevalence of EGFR amplification in GEA patients}

We evaluated EGFR copy number in four different cohorts: 1) a proprietary cohort (IRCC cohort) of 570 primary GEAs (real time PCR analysis); 2) the Foundation Medicine Inc. (FMI) dataset of 4337 gastric and 5060 esophageal/gastroesophageal junction [GEJ] adenocarcinomas (comprehensive genomic profiling); 3) the subgroup of 214 patients with esophageal or GEJ adenocarcinoma enrolled in the COG trial (NCT01243398) of second-line gefinitib versus placebo(10) (fluorescence in-situ hybridization, FISH); 4) the Fondazione IRCCS Istituto Nazionale dei Tumori (INT) of Milan dataset of 206 mGEA patients (in-situ hybridization, SISH). In the IRCC cohort we identified 44 primary tumors (7.8\%) with EGFR CNG ( $\geq 4$ gene copies), with 10 of them $(1.8 \%$ of all samples) bearing $>8$ gene copies (the suggested threshold of biologically meaningful amplification in the HER2 and MET context (30)) and 8 of them (1.4\% of all samples) bearing a heterogeneous $E G F R$ amplification (one tumor area $>8$ copies and one tumor area $\leq 8$ copies) (Figure 1 and Supplementary Table 1). In the FMI dataset 3.4\% of gastric and $7.6 \%$ of esophageal carcinomas showed EGFR amplification equal or higher than 8 copies, while in the COG and INT datasets the frequencies of EGFR amplification were $7.0 \%$ and $4.9 \%$, respectively (Figure 1). In both COG and INT cohorts, no significant association between EGFR amplification and baseline clinic-pathological characteristics was observed (Supplementary Table 2 and 3).

\section{EGFR amplification drives aggressiveness and poor prognosis in gastroesophageal adenocarcinomas}

To investigate if EGFR amplification is associated with poor prognosis of gastroesophageal adenocarcinomas, we took advantage of a cohort of pre-treated patients with esophageal and GEJ adenocarcinomas enrolled in the COG trial and randomized to placebo (10). Among 102 cases with available EGFR FISH status, patients with EGFR amplification had a significantly inferior median 
OS compared to those without EGFR amplification (3.1 versus 3.5 months; $\mathrm{HR}=1.23,95 \% \mathrm{CI}$ : 1.03-1.48; p=0.026; Figure 2A, left panel). All patients with EGFR amplified tumors died within 4 months.

Similarly, when focusing on the INT dataset, patients with EGFR amplification had inferior median OS as compared to those with EGFR SISH negative tumors (17.0 versus 18.9 months; HR=1.95, 95\% CI: 0.90-4.21; $\mathrm{p}=0.083$; Figure 2A, right panel). These results have been also confirmed in primary gastric tumors analyzing the TCGA data, in which tumor EGFR amplification correlated with significantly inferior OS and DFS (Figure 2B).

\section{Activity of EGFR inhibitors in patients with EGFR amplified metastatic gastric cancer and landscape of primary treatment resistance}

To determine whether patients with EGFR amplified mGEA may respond to EGFR inhibitors and to eliminate the potentially confounding effect of the combination with chemotherapy, we focused on patients with EGFR amplified mGEA treated at INT with the anti-EGFR monoclonal antibody panitumumab as single-agent after failure of standard treatment options. Three patients with EGFR amplification confirmed by SISH were identified (Supplementary Figure 1A); their molecular profile is summarized in Supplementary Figure 1B and their clinical history is reported in Figure 3. Briefly, INT\#1 patient had KRAS co-amplified mGEA and showed progressive disease (PD) at the first radiological re-assessment; INT\#2 had no co-occurring alterations in HER2, MET, KRAS or PIK3CA and showed a partial response (PR) lasting 6 months; INT\#3 had co-occurring heterogeneous KRAS amplification and showed a PR lasting only 10 weeks and followed by rapid clinical progression and death.

To verify if RTK pathway activation is associated with EGFR inhibitor resistance in GEA, we investigated the relationship between RTK copy number gain and survival following treatment with gefitinib in 12 EGFR FISH positive gastroesophageal adenocarcinomas (7 with amplification and 5 with high polysomy) of the COG trial. All 12 tumors analyzed had copy number gain (defined as $\geq 4$ 
gene copies) of at least one RTK (HER2, HER3, HER4, MET, FGFR1, FGFR2, FGFR3, FGFR4, IGF1R, PDGFR2,VEGFR1, VEGR2 and VEGFR3). We found a significant inverse correlation between the extent of co-amplification of the RTKs and OS (Figure 4A). This observation of shorter survival following gefitinib treatment with activation of RTKs other than EGFR suggests optimizing inhibition of downstream signal transduction pathways could produce durable clinical responses.

To investigate the prevalence of potential genetic predictors of primary resistance to anti-EGFR treatment, we interrogated the TCGA dataset for the presence of resistance alterations included in our previously published AMNESIA panel (18) among cases with EGFR amplification and showed the co-occurrence of other genomic events in $53 \%$ of samples (Suppl. Figure 2). Finally, since the available in-silico datasets mainly represent a collection of primary GEAs, we investigated the prevalence of AMNESIA panel alterations in the 534 samples from EGFR amplified mGEA patients included in the FMI dataset. This analysis showed the co-occurrence of other genomic events of interest in 186 (35\%) samples (Figure 4B).

\section{Dual EGFR blockade is the most effective treatment of EGFR-amplified PDXs}

Future trials might be prompted to re-assess the role of anti-EGFR MoAbs and TKI, either as monotherapy or in combination, in GEA molecularly selected patients. As already shown for dual HER2 blockade (trastuzumab plus pertuzumab or lapatinib) in HER2-positive breast and colorectal cancer (31-33), and despite the partially negative phase III data recently reported with this strategy in HER2-positive gastric cancer (34), dual EGFR blockade strategies with an anti-EGFR MoAb plus a TKI may be more effective than each drug as monotherapy.

A large series of human cancer specimens transplanted into mice (Patient-Derived Xenogratfs, PDXs) produce a study population that can be randomized for prospective treatment with targeted agents and so provide a strong strategy to perform precision medicine preclinical studies. This approach brings together the plasticity of preclinical analysis with the informative value of 
population-based studies. From 570 gastric carcinoma samples (IRCC cohort), we generated a multi-level platform of GEA models, comprising 151 PDXs, primary cell lines and organoids (22). Despite conflicting evidence on the CNG threshold clearly defining gene amplification, preclinical and clinical data obtained from GEA displaying HER2 or MET amplification suggested that the clinically relevant threshold is higher than 8 gene copies $(30,35)$. Eleven PDXs harbored at least 4-8 EGFR copies and 4 PDXs > 8 EGFR copies (Suppl. Figure 3A: GTR0060: 240 EGFR copies; GTR0078: 700 copies; GTR0110: 12 copies; GTR0511: 80 copies). These 4 models did not bear any other RTKs/KRAS CNV >8 copies (data not shown). SISH analysis and immunohistochemistry confirmed uniform EGFR amplification and expression (Suppl. Figure 3B). These PDX models were expanded to generate cohorts of mice, in order to evaluate the efficacy of the EGFR moAb cetuximab and the TKI erlotinib (EGFR-selective) and lapatinib (dual EGFR/HER2 inhibitor), as well as the combination of the moAb with a TKI. The original tumors were serially passaged in vivo until six tumor-bearing animals were produced per experimental group. When xenografts reached an average volume of $\sim 250 \mathrm{~mm}^{3}$, mice were randomized into six independent treatment cohorts: (i) vehicle (placebo); (ii) cetuximab; (iii) erlotinib; (iv) lapatinib; (v) cetuximab + erlotinib; (vi) cetuximab + lapatinib. Tumor response was evaluated according to RECIST-like Criteria (see Methods and Figure Legends).

As shown in Figure 5A, the GTR0060 PDX (240 EGFR copies) did not exhibit response to either of the TKIs used as monotherapy, but showed partial response (PR) upon cetuximab treatment. Notably, both the combo (cetuximab + TKI) treatments resulted in a complete response (CR). Interestingly, in 4 out of 6 mice in the combo arms, including 3/3 mice treated with erlotinib + cetuximab, the tumor mass did not reappear even after more than two months of drug removal (Figure 5B). Improved efficacy of the combo treatment was observed at long term also in a second model, GTR0110, characterized by a lower EGFR CNG (12 copies), uniformly distributed among tumor cells (Suppl. Figure 3B). While neither erlotinib nor lapatinib resulted in a clinical response and cetuximab conferred disease stabilization, cetuximab plus TKI combination treatment resulted 
in a PR (Figure 5C). Moreover, at the end of the experiment, the tumor volume was significantly reduced in mice treated with the combination compared with those treated with the moAb alone. The xenotrial performed in the GTR0511 PDX (80 EGFR copies) cohort also showed response to anti-EGFR treatment. Even though neither cetuximab nor lapatinib monotherapies were effective, their combination resulted in a relevant response. Interestingly, in this PDX erlotinib was the only effective monotherapy (Figure 5D). To investigate the reason of the differential sensitivity of GTR0511 to erlotinib, we analyzed Whole Exome Sequence data but we did not detect EGFR alterations (data not shown). On the contrary, RNAseq analysis revealed a ten-fold decrease of the number of reads covering the last portion of the receptor (from exon 26 until the end of the mRNA, Suppl. Figure 4A). This resulted in the presence of an EGFR protein isoform lacking the C-terminal domain, together with an EGFR full length protein. As Kovacs et al. (36) showed that the loss of this portion of the tail, containing Y1068, determines a strong decrease in receptor activation, we immunoprecipitated (with an antibody directed against the EGFR extracellular portion) EGFR from organoids derived from the three PDXs. As shown in Suppl. Fig. 4B, in GTR0511 EGFR displayed only a modest activation, in spite of the high amount of the expressed protein, meaning that the ratio between phosphorylated/unphosphorylated receptor is much lower in GTR0511 compared to the other amplified models. As predicted by in silico data, two phosphorylated bands were detected only in GTR0511, and they were both effectively inhibited by erlotinib. Finally, stronger downstream signal blockade in GTR0511 vs GTR0110 and GTR0060 was seen in total cell lysates derived from the same organoids. In agreement with previously published data (36) we thus hypothesize that the lack of the EGFR C-terminal tail in GTR0511 can be responsible of its decreased activation and increased sensitivity to erlotinib treatment..

To investigate which pathways were inactivated by the different drugs/drug combinations in cases in which the combo resulted in a strongly enhanced response, we took advantage of PDX-derived primary cells in which EGFR amplification was maintained (Suppl. Figure 5A). Primary cells were treated with cetuximab, erlotinib and lapatinib, alone or in combination. Western blot analysis 
showed that while lapatinib and erlotinib only slightly affected activation of downstream transducers such as AKT, MAPK and S6 (evaluated as read out of the PI3K, RAS/MAPK and mTOR pathways, respectively), a partial inhibition was induced by cetuximab. Interestingly, both the dual combinations resulted in a strong inhibition of signal transduction (Figure 5E). Phosphoarray analysis of cellular kinases and RTKs confirmed these results but did not identify any other kinase specifically inhibited by the combo treatments (Suppl. Figure 5B). These in vitro data strongly support the results we obtained in the in vivo experiments where cetuximab induced SD while the two combos resulted in a complete and durable response. It is thus likely that when EGFR activation is exceptionally intense, the dual blockade with TKI+cetuximab is needed to improve the response.

\section{TSC2 inactivation is a mechanism of resistance to EGFR targeted therapies.}

We performed a preclinical trial, similar to those previously described, using the GTR0078 PDX harboring 700 EGFR copies (Suppl. Figure 3). Despite the very high level of EGFR amplification we did not observe response to the TKIs, nor to cetuximab or cetuximab + TKI combos (Figure 6A). To understand the molecular basis for the observed resistance, we sequenced the tumor DNA and detected several genomic alterations; among these, we observed a fraction of EGFR gene copies displaying a deletion at the 5' gene portion, thus coding for a protein lacking the extracellular portion (Suppl. Figure 6A). Moreover, we also observed two missense TSC2 mutations (p.M1300V and p.R1438Q), with an allelic frequency of 0.463 and 0.539 , respectively (Figure 6B). The TSC2 protein forms a complex with TSC1, a critical negative regulator of mTORC1 (mammalian target of rapamycin complex 1) which controls anabolic processes to promote cell growth (37-39). TSC2 inactivation (due to homozygous mutations or gene loss), results in mTOR increased activation (40). Interestingly, when we interrogated cBioPortal for the possible cooccurrence of EGFR and TSC2 functional genomic alterations in six gastric cancer datasets (4,4145), we found a significant correlation (Suppl. Figure 6B). Moreover, alterations in the mTOR 
pathway co-occurrent with EGFR CNG have been identified in the FMI dataset as well, although co-occurrence with EGFR amplification was uncommon (Suppl. Figure 6C).

To support the causative role of TSC2 in EGFR target therapy resistance, we silenced TSC2 in OE21 cells, harboring EGFR gene amplification (46). In in vitro experiments, upon TSC2 silencing, we observed the constitutive activation of the mTOR pathway, revealed by the activation of the downstream transducer S6, which was maintained even in the presence of anti-EGFR treatment (Suppl. Figure 7A). To validate these data in vivo, we transduced OE21 cells with either Ctrl shRNA (shC) or a pool of TSC2 shRNAs and we injected them in immunocompromised mice. As shown in Suppl. Figure Fig. 7B, shC mice underwent tumor regression in response to EGFR blockade, while partially TSC2 silenced tumors experienced only disease stabilization, reinforcing the idea that TSC2 silencing impairs the response to anti-EGFR therapy.

We thus wondered if treatment of GTR0078 tumors with a mTOR inhibitor (such as everolimus) could restore sensitivity to EGFR inhibitors. While treatment with everolimus alone did not show any clinical efficacy (Suppl. Figure 6D), the combination of everolimus with erlotinib resulted in a significant clinical response (Figure 6C). Experiments performed in PDX-derived cells showed that while treatment of GTR0078 cells with either EGFR inhibitors or everolimus was unable to block mTOR activation, the association of the two drugs resulted in a sustained inhibition of the pathway. Indeed, only the concomitant inhibition of the EGFR and mTOR pathway inactivated the downstream transducer S6 kinase (Figure 6D). 


\section{DISCUSSION}

In unselected patients with advanced gastric/esophageal adenocarcinoma, the addition of an anti EGFR antibody to first-line standard chemotherapy failed to show a significant survival benefit in two randomized clinical trials $(8,9)$. Similar negative results were also observed when the small molecule TK inhibitor gefitinib was compared to placebo from the second-line setting and beyond (10). Sporadic responses to EGFR inhibitors observed in these trials, however, lead several researchers to postulate the existence of a subset of metastatic patient with $E G F R$-addicted tumors, potentially vulnerable to EGFR blockade (13). The amplification of the EGFR gene is found in 3$5 \%$ of primary GEA tumors $(4,6)$ and highly correlates with poor prognosis (47). By exploiting four different datasets, we have shown here that EGFR amplification has similar prevalence and is associated with poorer survival in the metastatic setting. This was also confirmed in the nonmetastatic setting, analyzing the TCGA data. In a pre-specified exploratory analysis of one of those data sets, the COG trial randomizing 209 chemo-resistant metastatic patients to gefitinib or placebo (10), EGFR amplification is a positive predictive marker for EGFR targeting, whereas a smaller advantage is observed in patients with chromosome 7 polysomy (13). Response to the anti-EGFR moAb cetuximab, used alone or in combination with chemotherapy, was reported in a small set of seven EGFR amplified patients; albeit the role of the cytotoxic backbone contribution cannot be ruled out in three responders, one response was induced by EGFR blockade alone (48). Such results clearly mirror those achieved in patients receiving panitumumab monotherapy by our study. All together these observations suggest that $E G F R$ is an oncogenic driver, with potentially exquisite sensitivity to EGFR targeting drugs, in a small but clinically consistent subgroup of GEAs. On the other hand, in these EGFR-amplified tumors, we observed the presence of selected co-occurring driver alterations. Specifically $M E T / H E R 2 / K R A S$ co-amplifications and KRAS/PK3CA/PTEN comutations were identified in $53 \%$ and $35 \%$ of patients in the EGFR amplified subgroups included in the TCGA and FMI datasets, respectively; this result highlights that only a subset of patients with EGFR amplified gastroesophageal cancer may significantly benefit from single-agent anti-EGFR 
therapy. Here, we have for the first time functionally identified TSC2 mutations as a potential new mechanism conferring resistance to EGFR inhibition in GEAs. TSC2 is a GTPase-activating protein, whose loss or inactivating mutation result in the constitutive load of Rheb with GTP and activation of mTORC signaling (39). Interestingly, according to cBioPortal, TSC1/TSC2 mutations are significantly associated with EGFR amplification (but not with other RTKs) in GEAs, possibly indicating that mTORC constitutive activation can sustain the oncogenic role of EGFR. Our preclinical trial in an EGFR amplified/TSC2 mutated GEA PDX confirms this hypothesis. The pharmacological inhibition of TSC2-sustained mTORC activation by everolimus, a clinical grade small molecule mTOR inhibitor, overcame primary resistance and restored sensitivity to EGFR inhibition. Our data are reinforced by a recently published paper from Arteaga and coll. (49), in which they showed that hyperactivation of the mTORC pathway drives resistance to therapies targeting another member of the HER family, namely HER2, in HER2-mutant breast cancer. In their work, similarly to what we have observed, the combination of the TORC1 inhibitor everolimus and neratinib overcame resistance.

Resistance is a common occurrence of RTK inhibition across diseases, targets and drugs. Several cell autonomous mechanisms sustaining resistance to driver RTKs have been identified so far, including mutations of the target itself, activation of downstream transducers, activation of parallel pathways and transdifferentiation. Moreover, in many cases the amplified RTK is not located in the natural genomic site, but it is rather extrachromosomal. This results in a mechanism favoring rapid adaptation of cancer cells to environmental changes. Indeed, as extrachromosomal DNA lacks centromeres, it is unequally segregated during cell division, leading to increased tumor heterogeneity and different cellular fitness in diverse contexts. Cancer cells in which oncogenes are extrachromosomal can thus become resistant to RTK inhibitors either by increasing the number of gene copies (thus titrating the amount of the available inhibitor) or by progressively decreasing the number of gene copies. Both the mechanisms are sustained by experimental data. For example, Nathanson et al. (50) showed that glioblastoma cells can become resistant to erlotinib, eliminating 
extrachromosomal copies of the mutant EGFR gene. This "adaptation" to the treatment can be acquired and expanded along tumor evolution, enabling tumors to maintain their intratumoral heterogeneity. In previous works $(51,52)$ we have shown that in MET hyper amplified gastric cancer cells (where the amplified gene was extrachromosomal) resistance was due to further acquisition of gene copies; this resulted in an amount of activated receptor overcoming the inhibitory ability of the drugs at tolerable doses.

To bypass primary and prevent secondary resistance to EGFR-targeted drugs in EGFR amplified GEAs we leveraged our large platform of 151 primary GEAs patient-derived mouse avatars (22), enriched for 15 cases with EGFR gene copy gain, including 4 avatars with more than $8 E G F R$ copies (confirmed as amplified -i.e. non polysomic- by silver in situ hybridization). EGFR inhibition, in absence of chemotherapy, resulted in a clinical response in three out of four cases. Notably, one of these cases featured 12 EGFR copies, a range of amplification that is just above the threshold (8 copies) considered biologically relevant and that has not been investigated previously (48). Interestingly, a complete response was achieved only in the PDX with the highest EGFR CNG, suggesting that a higher level of gene amplification may be associated with a greater magnitude of treatment benefit, as it is known for HER2-amplified GEA and breast cancer $(30,53)$.

The pharmacological space of EGFR targeted drugs is well populated by antibodies and small molecule TKIs, both experimental and approved for use in clinically diverse settings $(54,55)$.

In our preclinical trials in EGFR-amplified GEA avatars we compared the efficacy of randomly allocated TKIs and cetuximab, delivered as single agent or in combinations. Erlotinib and cetuximab showed single agent excellent activity in one and two models respectively, while in a third model cetuximab treatment resulted in disease stabilization. Importantly however, the dual EGFR blockade resulted in a sustained significant response in all three models suggesting that a strong inhibition of the downstream transducers is needed to eradicate the disease.

In conclusion, our study further corroborates EGFR amplification as an actionable therapeutic target in GEA, demonstrates that a dual EGFR blockade may be needed to maximize the therapeutic 
efficacy, and identifies potential mechanisms of primary resistance, specifically the mTORC pathway, paving the way for experimentally driven clinical trials. In fact, the next generation clinical trial landscape in EGFR amplified GEAs may not be at a dead end. The combination of lapatinib and cetuximab has already been proven safe in a phase 1 trial (56), potent secondgeneration antibodies mixtures against different, non-overlapping epitopes of EGFR - such as Sym004 and MM-151 (57,58) - are into clinical development and the TORC pathway is targetable with commercially available drugs. Given the diversity of clinically relevant genomic alterations and lack of benefit from EGFR targeted therapies in unselected GEA populations, broad based genomic profiling is thus necessary to reliably detect EGFR gene amplification in addition to other potential drivers and mechanisms of resistance. 


\section{ACKNOWLEDGEMENTS}

We thank Dr. Adam Bass for performing sequencing analysis of IRCC samples; our colleagues of GIRCG ('Gruppo Italiano Ricerca Carcinoma Gastrico') for their support; G. Manessi for experimental help; B.Martinoglio, M.Buscarino and M.Montone for technical support with Real Time PCR and Cell-ID; I.Sarotto, D.Balmativola, E.Maldi, M.Volante, A.Rigutto for pathological analysis; animal facility employees; L.Trusolino and A.Bertotti for helpful scientific discussion. SG, SC are EurOPDX Consortium members.

\section{GRANT SUPPORT}

This work was funded by the Italian Association for Cancer Research (AIRC), IG 20210 to SG, 21770 to S. Corso and IG 23624 to F. Pietrantonio; Fondazione Piemontese per la Ricerca sul Cancro (FPRC, ONLUS) 5X1000 Min. Salute 2013 to A.Sottile; FPRC 5X1000 2014 Min. Salute to SG and to A.Sapino; FPRC 5X1000 2015 Min. Salute to SG and to A. Sapino; FPRC 5X1000 2015 Min. Salute "Strategy" to SG; Ricerca Corrente 2019, Min. Salute to A.Sapino.

\section{AUTHOR CONTRIBUTIONS}

SG, SC, FP and MA conceived and supervised the study, contributed to design the experiments, wrote the manuscript; MD, RR, UF, SDP, GS, ER, GLB, SM, GDM, MB; SS, AS-B, FM, SC, MP, MDB provided patient material and data; C.Migliore, AP, DC, LDE, SD, DMR, SEB, SU performed experiments; C.Marchio', A.Sapino, AG performed the pathologic analysis; JL, SMA, JSR, BA, VAM and ABS provided data from FMI; A.Sottile provided technical support; ADS, ZM, BA, JSR, RP provided data from the COG study; S.Marsoni managed the GEA Study. All the authors revised the manuscript. 


\section{FIGURE LEGENDS}

Figure 1. EGFR copy number gain. The graphs illustrate the percentage of tumors displaying EGFR copy number gain (CNG) in four different cohorts. Real time PCR analysis of IRCC gastric/gastroesophageal junction (GEJ) adenocarcinomas displaying EGFR gain (4-8 copies or more than 8 copies) or heterogeneity (significantly different EGFR CNG in diverse analyzed samples from the same tumor, with one tumor sample $>8$ copies and one tumor sample $\leq 8$ copies). Comprehensive genomic profiling of FMI gastric and esophageal/GEJ cases, FISH analysis of COG esophageal/GEJ cases and SISH analysis of INT gastric/GEJ adenocarcinomas.

Figure 2. Survival analysis of patients with EGFR CNG. (A) The graphs show the cumulative survival (Cum Survival) of patients of the COG (left) and INT (right) cohorts related to EGFR CNG. (B) The graphs show the overall survival (left) and the disease-free survival (right) of patients of the gastroesophageal TCGA dataset, related to EGFR CNG.

Figure 3. Clinical history of patients treated with EGFR-targeted drugs. Summarized clinical course of INT patients with EGFR CNG. Red-lined boxes indicate periods of administration of the indicated therapeutic agents. Blue vertical lines indicate timing of tumor specimen acquisition from surgical procedures or biopsies, as well as dates of tumor assessment by CT scan. PD, progressive disease; SD, stable disease, according to RECIST 1.1. EOX: epirubicin, oxaliplatin and capecitabine; 5FU: 5 fluorouracil; TCF: docetaxel, carboplatin and 5-fluorouracil; FOLFIRI: folinic acid, 5-fluorouracil, irinotecan; XELOX: capecitabine and oxaliplatin; CCDP: cisplatin, vinorelbine, ifosfamide and epirubicin; OGD: oesophago-gastro-duodenoscopy.

Figure 4. RTK/KRAS pathway activation in EGFR amplified cases. (A) The scatter plot shows a significant inverse correlation between the extent of RTKs co-amplification (HER2, HER3, HER4, MET, FGFR1, FGFR2, FGFR3, FGFR4, IGF1R, PDGFR2, VEGFR1, VEGR2 and VEGFR3 $\geq 4$ gene copies) and OS in 12 EGFR FISH positive GEAs treated with gefitinib in the COG trial. Red 
dots indicate cases with high polisomy; blue dots represent cases with EGFR amplification. (B) The graph shows the co-occurrence of EGFR amplification and genomic events affecting the RTK/KRAS pathway in EGFR amplified GEA tumors in the FMI dataset.

Figure 5. Dual EGFR blockade is the most effective treatment in EGFR-amplified PDXs Tumor growth curves in mice cohorts derived from GTR0060 (A), GTR0110 (C) and GTR0511 (D) patients treated with the EGFR inhibitors cetuximab (CETUX), erlotinib (ERL), lapatinib (LAP), alone or in combination, as indicated. The red lines indicate the day when treatment was started. The response in mice has been evaluated using RECIST 1.1-like criteria, i.e. progressive disease (PD): $\geq 35 \%$ increase from baseline; partial response (PR): $\geq 50 \%$ reduction from baseline; stable disease (SD): intermediate variations from baseline. (B) Spaghetti plot illustrating drug response in the xenotrial performed on the cohort of mice derived from PDX GTR0060. Individual lines represent, for each mouse, the percentage variation in tumor burden, from treatment start (day 0). Blue lines: CETUX + LAP treated mice; red lines: CETUX + ERL treated mice. Dashed line indicates treatment stop. (E) Western blot analysis of the activation state of EGFR and its downstream targets (AKT, MAPK and S6) in GTR0060 tumor-derived cells treated with the indicated drugs/drug combinations. Actin was used as loading control. Statistical significance is indicated $* *<0.01 ; * * *<0.001$

\section{Figure 6. TSC2 inactivation is a mechanism of resistance to EGFR targeted therapies.}

(A) Tumor growth curves in the mice cohorts derived from GTR0078 treated with the EGFR inhibitors cetuximab (CETUX), erlotinib (ERL), lapatinib (LAP), alone or in combination, as indicated. The arrow indicates the day when treatment was started. (B) The table shows the two TSC2 mutations identified in GTR0078 PDX. (C) Tumor growth curves in the mice cohorts derived from GTR0078 treated with erlotinib (ERL) or the combo erlotinib + everolimus (ERL+ EVEROL). The red line indicates the day when treatment was started. (D) Western blot analysis of the activation state of EGFR and its downstream targets (AKT, MAPK and S6) in GTR0078 tumor- 
derived cells treated with the indicated drugs/drug combinations. Actin was used as loading control. Statistical significance is indicated $* * *<0.001$ 


\section{REFERENCES}

1. Ferlay J, Soerjomataram I, Dikshit R, Eser S, Mathers C, Rebelo M, et al. Cancer incidence and mortality worldwide: Sources, methods and major patterns in GLOBOCAN 2012. Int J Cancer 2015;136(5):E359-86 doi 10.1002/ijc.29210.

2. Corso S, Giordano S. How Can Gastric Cancer Molecular Profiling Guide Future Therapies? Trends Mol Med 2016;22(7):534-44 doi 10.1016/j.molmed.2016.05.004.

3. Raimondi A, Nichetti F, Peverelli G, Di Bartolomeo M, De Braud F, Pietrantonio F. Genomic markers of resistance to targeted treatments in gastric cancer: potential new treatment strategies. Pharmacogenomics 2018;19(13):1047-68 doi 10.2217/pgs-2018-0077.

4. Network CGAR. Comprehensive molecular characterization of gastric adenocarcinoma. Nature 2014;513(7517):202-9 doi 10.1038/nature13480.

5. Cristescu R, Lee J, Nebozhyn M, Kim KM, Ting JC, Wong SS, et al. Molecular analysis of gastric cancer identifies subtypes associated with distinct clinical outcomes. Nat Med 2015;21(5):449-56 doi $10.1038 / \mathrm{nm} .3850$.

6. Schrock AB, Devoe CE, McWilliams R, Sun J, Aparicio T, Stephens PJ, et al. Genomic Profiling of Small-Bowel Adenocarcinoma. JAMA Oncol 2017;3(11):1546-53 doi 10.1001/jamaoncol.2017.1051.

7. Pirker R, Pereira JR, Szczesna A, von Pawel J, Krzakowski M, Ramlau R, et al. Cetuximab plus chemotherapy in patients with advanced non-small-cell lung cancer (FLEX): an open-label randomised phase III trial. Lancet 2009;373(9674):1525-31 doi 10.1016/S0140-6736(09)60569-9.

8. Lordick F, Kang YK, Chung HC, Salman P, Oh SC, Bodoky G, et al. Capecitabine and cisplatin with or without cetuximab for patients with previously untreated advanced gastric cancer (EXPAND): a randomised, open-label phase 3 trial. Lancet Oncol 2013;14(6):490-9 doi 10.1016/S14702045(13)70102-5.

9. Waddell T, Chau I, Cunningham D, Gonzalez D, Okines AF, Frances A, et al. Epirubicin, oxaliplatin, and capecitabine with or without panitumumab for patients with previously untreated advanced 
oesophagogastric cancer (REAL3): a randomised, open-label phase 3 trial. Lancet Oncol 2013;14(6):481-9 doi 10.1016/S1470-2045(13)70096-2.

10. Dutton SJ, Ferry DR, Blazeby JM, Abbas H, Dahle-Smith A, Mansoor W, et al. Gefitinib for oesophageal cancer progressing after chemotherapy (COG): a phase 3, multicentre, double-blind, placebo-controlled randomised trial. Lancet Oncol 2014;15(8):894-904 doi 10.1016/S14702045(14)70024-5.

11. Zhang L, Yang J, Cai J, Song X, Deng J, Huang X, et al. A subset of gastric cancers with EGFR amplification and overexpression respond to cetuximab therapy. Sci Rep 2013;3:2992 doi 10.1038/srep02992.

12. Huang J, Fan Q, Lu P, Ying J, Ma C, Liu W, et al. Icotinib in Patients with Pretreated Advanced Esophageal Squamous Cell Carcinoma with EGFR Overexpression or EGFR Gene Amplification: A Single-Arm, Multicenter Phase 2 Study. J Thorac Oncol 2016;11(6):910-7 doi 10.1016/j.jtho.2016.02.020.

13. Petty RD, Dahle-Smith A, Stevenson DAJ, Osborne A, Massie D, Clark C, et al. Gefitinib and EGFR Gene Copy Number Aberrations in Esophageal Cancer. J Clin Oncol 2017;35(20):2279-87 doi $10.1200 / J C O .2016 .70 .3934$

14. Apicella M, Migliore C, Capelôa T, Menegon S, Cargnelutti M, Degiuli M, et al. Dual MET/EGFR therapy leads to complete response and resistance prevention in a MET-amplified gastroesophageal xenopatient cohort. Oncogene 2016 doi 10.1038/onc.2016.283.

15. Miyoshi $\mathrm{H}$, Stappenbeck TS. In vitro expansion and genetic modification of gastrointestinal stem cells in spheroid culture. Nat Protoc 2013;8(12):2471-82 doi 10.1038/nprot.2013.153.

16. Sigismund S, Algisi V, Nappo G, Conte A, Pascolutti R, Cuomo A, et al. Threshold-controlled ubiquitination of the EGFR directs receptor fate. EMBO J 2013;32(15):2140-57 doi 10.1038/emboj.2013.149. 
17. Corso S, Migliore C, Ghiso E, De Rosa G, Comoglio PM, Giordano S. Silencing the MET oncogene leads to regression of experimental tumors and metastases. Oncogene 2008;27(5):684-93 doi 10.1038/sj.onc.1210697.

18. Pietrantonio F, Fucà G, Morano F, Gloghini A, Corso S, Aprile G, et al. Biomarkers of Primary Resistance to Trastuzumab in HER2-Positive Metastatic Gastric Cancer Patients: the AMNESIA CaseControl Study. Clin Cancer Res 2018;24(5):1082-9 doi 10.1158/1078-0432.CCR-17-2781.

19. Corso S, Cargnelutti M, Durando S, Menegon S, Apicella M, Migliore C, et al. Rituximab Treatment Prevents Lymphoma Onset in Gastric Cancer Patient-Derived Xenografts. Neoplasia 2018;20(5):44355 doi 10.1016/j.neo.2018.02.003.

20. Bertotti A, Migliardi G, Galimi F, Sassi F, Torti D, Isella C, et al. A molecularly annotated platform of patient-derived xenografts ("xenopatients") identifies HER2 as an effective therapeutic target in cetuximab-resistant colorectal cancer. Cancer Discov 2011;1(6):508-23 doi 10.1158/2159-8290.CD11-0109.

21. Pectasides E, Stachler MD, Derks S, Liu Y, Maron S, Islam M, et al. Genomic Heterogeneity as a Barrier to Precision Medicine in Gastroesophageal Adenocarcinoma. Cancer Discov 2018;8(1):37-48 doi 10.1158/2159-8290.CD-17-0395.

22. Corso S, Isella C, Bellomo SE, Apicella M, Durando S, Migliore C, et al. A comprehensive PDX gastric cancer collection captures cancer cell intrinsic transcriptional MSI traits. Cancer Res 2019 doi 10.1158/0008-5472.CAN-19-1166.

23. Frampton GM, Fichtenholtz A, Otto GA, Wang K, Downing SR, He J, et al. Development and validation of a clinical cancer genomic profiling test based on massively parallel DNA sequencing. Nat Biotechnol 2013;31(11):1023-31 doi 10.1038/nbt.2696.

24. Van Loo P, Nordgard SH, Lingjærde OC, Russnes HG, Rye IH, Sun W, et al. Allele-specific copy number analysis of tumors. Proc Natl Acad Sci U S A 2010;107(39):16910-5 doi 10.1073/pnas.1009843107. 
25. Diskin SJ, Eck T, Greshock J, Mosse YP, Naylor T, Stoeckert CJ, et al. STAC: A method for testing the significance of DNA copy number aberrations across multiple array-CGH experiments. Genome Res 2006;16(9):1149-58 doi 10.1101/gr.5076506.

26. Morano F, Corallo S, Lonardi S, Raimondi A, Cremolini C, Rimassa L, et al. Negative Hyperselection of Patients With. J Clin Oncol 2019:JCO1901254 doi 10.1200/JCO.19.01254.

27. Conway T, Wazny J, Bromage A, Tymms M, Sooraj D, Williams ED, et al. Xenome--a tool for classifying reads from xenograft samples. Bioinformatics 2012;28(12):i172-8 doi 10.1093/bioinformatics/bts236.

28. Kim D, Langmead B, Salzberg SL. HISAT: a fast spliced aligner with low memory requirements. Nat Methods 2015;12(4):357-60 doi 10.1038/nmeth.3317.

29. Anders S, Pyl PT, Huber W. HTSeq--a Python framework to work with high-throughput sequencing data. Bioinformatics 2015;31(2):166-9 doi 10.1093/bioinformatics/btu638.

30. Gomez-Martin C, Plaza JC, Pazo-Cid R, Salud A, Pons F, Fonseca P, et al. Level of HER2 gene amplification predicts response and overall survival in HER2-positive advanced gastric cancer treated with trastuzumab. J Clin Oncol 2013;31(35):4445-52 doi 10.1200/JCO.2013.48.9070.

31. von Minckwitz G, Procter M, de Azambuja E, Zardavas D, Benyunes M, Viale G, et al. Adjuvant Pertuzumab and Trastuzumab in Early HER2-Positive Breast Cancer. N Engl J Med 2017;377(2):12231 doi 10.1056/NEJMoa1703643.

32. Baselga J, Cortés J, Kim SB, Im SA, Hegg R, Im YH, et al. Pertuzumab plus trastuzumab plus docetaxel for metastatic breast cancer. N Engl J Med 2012;366(2):109-19 doi 10.1056/NEJMoa1113216.

33. Swain SM, Baselga J, Kim SB, Ro J, Semiglazov V, Campone M, et al. Pertuzumab, trastuzumab, and docetaxel in HER2-positive metastatic breast cancer. N Engl J Med 2015;372(8):724-34 doi 10.1056/NEJMoa1413513.

34. Tabernero J, Hoff PM, Shen L, Ohtsu A, Shah MA, Cheng K, et al. Pertuzumab plus trastuzumab and chemotherapy for HER2-positive metastatic gastric or gastro-oesophageal junction cancer (JACOB): 
final analysis of a double-blind, randomised, placebo-controlled phase 3 study. Lancet Oncol 2018;19(10):1372-84 doi 10.1016/S1470-2045(18)30481-9.

35. Suda K, Murakami I, Katayama T, Tomizawa K, Osada H, Sekido Y, et al. Reciprocal and complementary role of MET amplification and EGFR T790M mutation in acquired resistance to kinase inhibitors in lung cancer. Clin Cancer Res 2010;16(22):5489-98 doi 10.1158/1078-0432.CCR10-1371.

36. Kovacs E, Das R, Wang Q, Collier TS, Cantor A, Huang $\mathrm{Y}$, et al. Analysis of the Role of the C-Terminal Tail in the Regulation of the Epidermal Growth Factor Receptor. Mol Cell Biol 2015;35(17):3083-102 doi 10.1128/MCB.00248-15.

37. Inoki K, Li Y, Zhu T, Wu J, Guan KL. TSC2 is phosphorylated and inhibited by Akt and suppresses mTOR signalling. Nat Cell Biol 2002;4(9):648-57 doi 10.1038/ncb839.

38. Tee AR, Fingar DC, Manning BD, Kwiatkowski DJ, Cantley LC, Blenis J. Tuberous sclerosis complex-1 and -2 gene products function together to inhibit mammalian target of rapamycin (mTOR)mediated downstream signaling. Proc Natl Acad Sci U S A 2002;99(21):13571-6 doi 10.1073/pnas.202476899.

39. Li Y, Corradetti MN, Inoki K, Guan KL. TSC2: filling the GAP in the mTOR signaling pathway. Trends Biochem Sci 2004;29(1):32-8 doi 10.1016/j.tibs.2003.11.007.

40. Chan JA, Zhang H, Roberts PS, Jozwiak S, Wieslawa G, Lewin-Kowalik J, et al. Pathogenesis of tuberous sclerosis subependymal giant cell astrocytomas: biallelic inactivation of TSC1 or TSC2 leads to mTOR activation. J Neuropathol Exp Neurol 2004;63(12):1236-42 doi 10.1093/jnen/63.12.1236.

41. Chen K, Yang D, Li X, Sun B, Song F, Cao W, et al. Mutational landscape of gastric adenocarcinoma in Chinese: implications for prognosis and therapy. Proc Natl Acad Sci U S A 2015;112(4):1107-12 doi 10.1073/pnas.1422640112. 
42. Guo YA, Chang MM, Huang W, Ooi WF, Xing M, Tan P, et al. Mutation hotspots at CTCF binding sites coupled to chromosomal instability in gastrointestinal cancers. Nat Commun 2018;9(1):1520 doi 10.1038/s41467-018-03828-2.

43. Wang K, Yuen ST, Xu J, Lee SP, Yan HH, Shi ST, et al. Whole-genome sequencing and comprehensive molecular profiling identify new driver mutations in gastric cancer. Nat Genet 2014;46(6):573-82 doi 10.1038/ng.2983.

44. Kakiuchi M, Nishizawa T, Ueda H, Gotoh K, Tanaka A, Hayashi A, et al. Recurrent gain-of-function mutations of RHOA in diffuse-type gastric carcinoma. Nat Genet 2014;46(6):583-7 doi 10.1038/ng.2984.

45. Wang K, Kan J, Yuen ST, Shi ST, Chu KM, Law S, et al. Exome sequencing identifies frequent mutation of ARID1A in molecular subtypes of gastric cancer. Nat Genet 2011;43(12):1219-23 doi 10.1038/ng.982.

46. Zhou J, Wu Z, Wong G, Pectasides E, Nagaraja A, Stachler M, et al. CDK4/6 or MAPK blockade enhances efficacy of EGFR inhibition in oesophageal squamous cell carcinoma. Nat Commun 2017;8:13897 doi 10.1038/ncomms13897.

47. Chen C, Yang JM, Hu TT, Xu TJ, Yan G, Hu SL, et al. Prognostic role of human epidermal growth factor receptor in gastric cancer: a systematic review and meta-analysis. Arch Med Res 2013;44(5):380-9 doi 10.1016/j.arcmed.2013.07.001.

48. Maron SB, Alpert L, Kwak HA, Lomnicki S, Chase L, Xu D, et al. Targeted Therapies for Targeted Populations: Anti-EGFR Treatment for. Cancer Discov 2018;8(6):696-713 doi 10.1158/21598290.CD-17-1260.

49. Sudhan DR, Guerrero-Zotano A, Won H, Ericsson PG, Servetto A, Huerta-Rosario M, et al. Hyperactivation of TORC1 Drives Resistance to the Pan-HER Tyrosine Kinase Inhibitor Neratinib in HER2-Mutant Cancers. Cancer Cell 2020;37(2):258-9 doi 10.1016/j.ccell.2020.01.010. 
50. Nathanson DA, Gini B, Mottahedeh J, Visnyei K, Koga T, Gomez G, et al. Targeted therapy resistance mediated by dynamic regulation of extrachromosomal mutant EGFR DNA. Science 2014;343(6166):72-6 doi 10.1126/science.1241328.

51. Cepero V, Sierra JR, Corso S, Ghiso E, Casorzo L, Perera T, et al. MET and KRAS gene amplification mediates acquired resistance to MET tyrosine kinase inhibitors. Cancer Res 2010;70(19):7580-90 doi 10.1158/0008-5472.CAN-10-0436.

52. Martin V, Corso S, Comoglio PM, Giordano S. Increase of MET gene copy number confers resistance to a monovalent MET antibody and establishes drug dependence. Mol Oncol 2014 doi 10.1016/j.molonc.2014.06.010

53. Veeraraghavan J, De Angelis C, Mao R, Wang T, Herrera S, Pavlick AC, et al. A combinatorial biomarker predicts pathologic complete response to neoadjuvant lapatinib and trastuzumab without chemotherapy in patients with HER2+ breast cancer. Ann Oncol 2019 doi 10.1093/annonc/mdz076.

54. Moradi-Kalbolandi S, Hosseinzade A, Salehi M, Merikhian P, Farahmand L. Monoclonal antibodybased therapeutics, targeting the epidermal growth factor receptor family: from herceptin to Pan HER. J Pharm Pharmacol 2018;70(7):841-54 doi 10.1111/jphp.12911.

55. Singh D, Attri BK, Gill RK, Bariwal J. Review on EGFR Inhibitors: Critical Updates. Mini Rev Med Chem 2016;16(14):1134-66 doi 10.2174/1389557516666160321114917.

56. Deeken JF, Wang H, Subramaniam D, He AR, Hwang J, Marshall JL, et al. A phase 1 study of cetuximab and lapatinib in patients with advanced solid tumor malignancies. Cancer 2015;121(10):1645-53 doi 10.1002/cncr.29224.

57. Kearns JD, Bukhalid R, Sevecka M, Tan G, Gerami-Moayed N, Werner SL, et al. Enhanced Targeting of the EGFR Network with MM-151, an Oligoclonal Anti-EGFR Antibody Therapeutic. Mol Cancer Ther 2015;14(7):1625-36 doi 10.1158/1535-7163.MCT-14-0772. 
Author Manuscript Published OnlineFirst on February 4, 2021; DOI: 10.1158/1078-0432.CCR-20-0121

Author manuscripts have been peer reviewed and accepted for publication but have not yet been edited.

58. Pedersen MW, Jacobsen HJ, Koefoed K, Hey A, Pyke C, Haurum JS, et al. Sym004: a novel synergistic anti-epidermal growth factor receptor antibody mixture with superior anticancer efficacy. Cancer Res 2010;70(2):588-97 doi 10.1158/0008-5472.CAN-09-1417. 


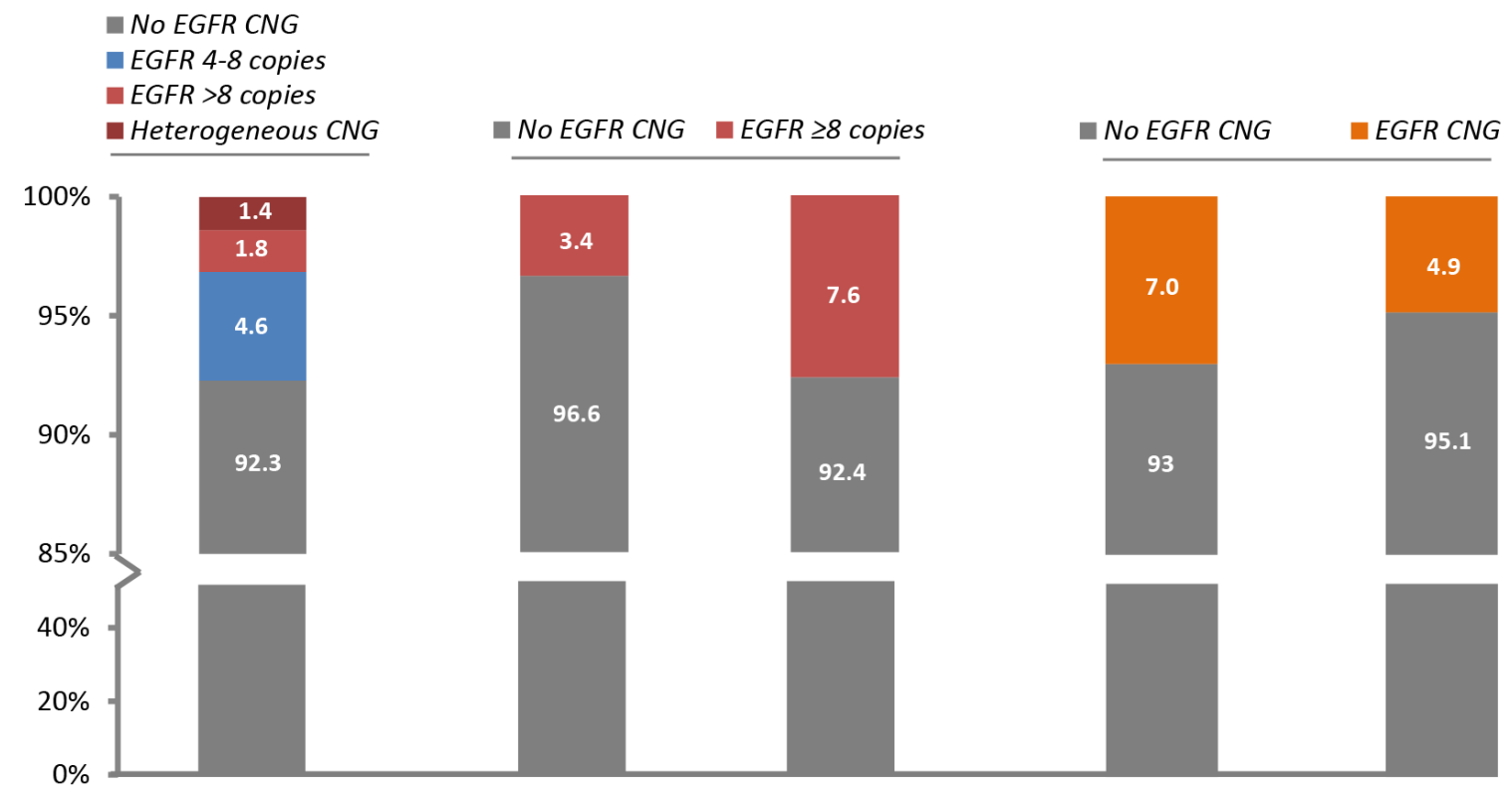

\begin{tabular}{|c|c|c|c|c|c|}
\hline \multirow{2}{*}{ COHORT NAME } & IRCC & \multicolumn{2}{|c|}{ FMI } & COG & INT \\
\hline SAMPLE TYPE & Gastric/GEJ & Gastric & Esophageal/GEJ & Esophageal/GEJ & Gastric/GEJ \\
\hline SAMPLE SIZE & $\mathrm{N}=\mathbf{5 7 0}$ & $\mathbf{N}=\mathbf{4 3 3 7}$ & $\mathbf{N}=5060$ & $\mathrm{~N}=\mathbf{2 1 4}$ & N=206 \\
\hline $\begin{array}{c}\text { EGFR CNG } \\
\text { DETECTION }\end{array}$ & qReal Time PCR & Genomic Profiling & Genomic Profiling & FISH & SISH \\
\hline
\end{tabular}


A

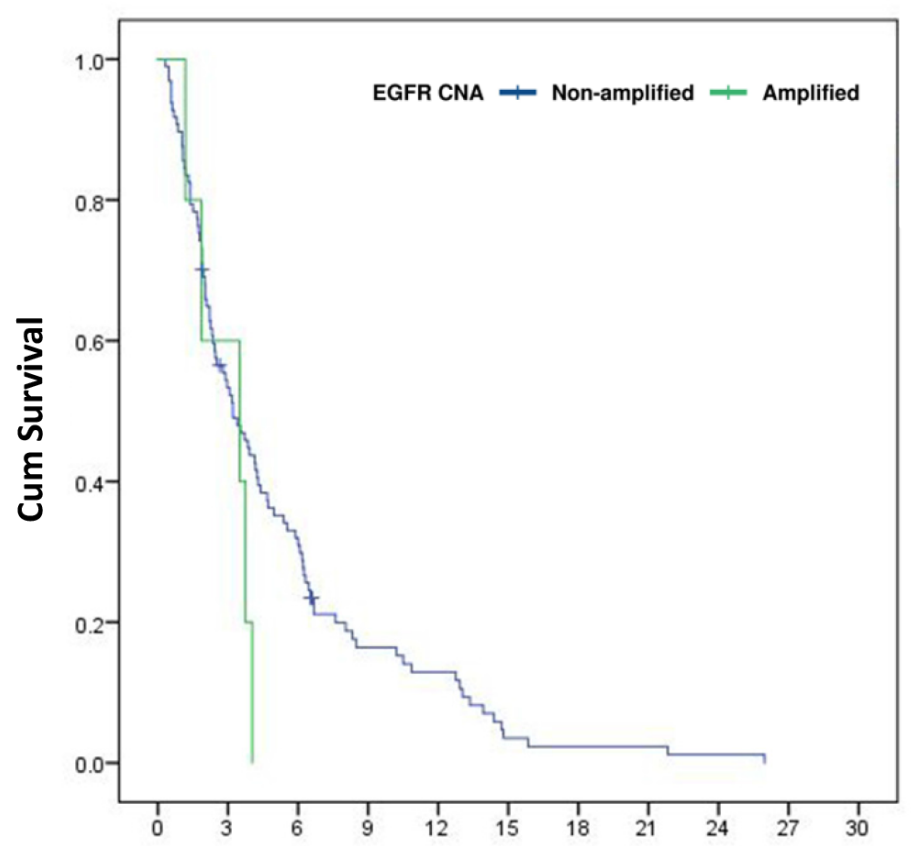

Overall Survival (months)

\section{Number at risk}

$\begin{array}{ccccccccccc}94 & 50 & 29 & 19 & 12 & 3 & 2 & 1 & 1 & 0 & 0 \\ 8 & 3 & 0 & 0 & 0 & 0 & 0 & 0 & 0 & 0 & 0\end{array}$

B

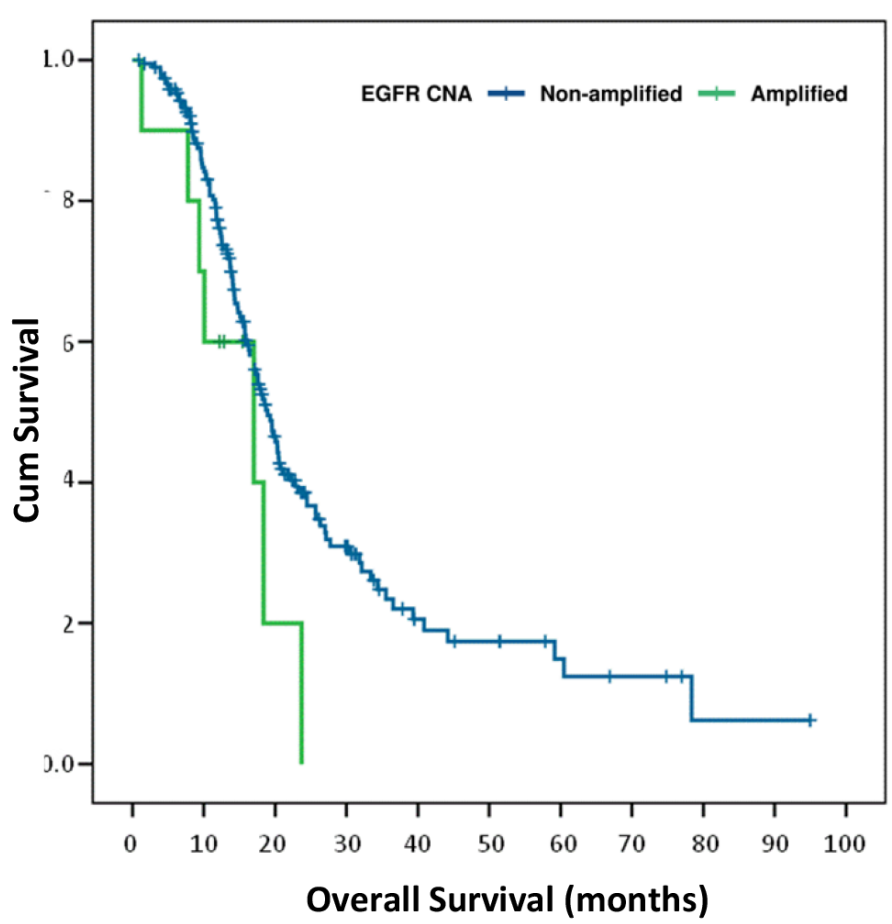

Number at risk

$\begin{array}{lllllllllll}10 & 7 & 1 & 0 & 0 & 0 & 0 & 0 & 0 & 0 & 0\end{array}$

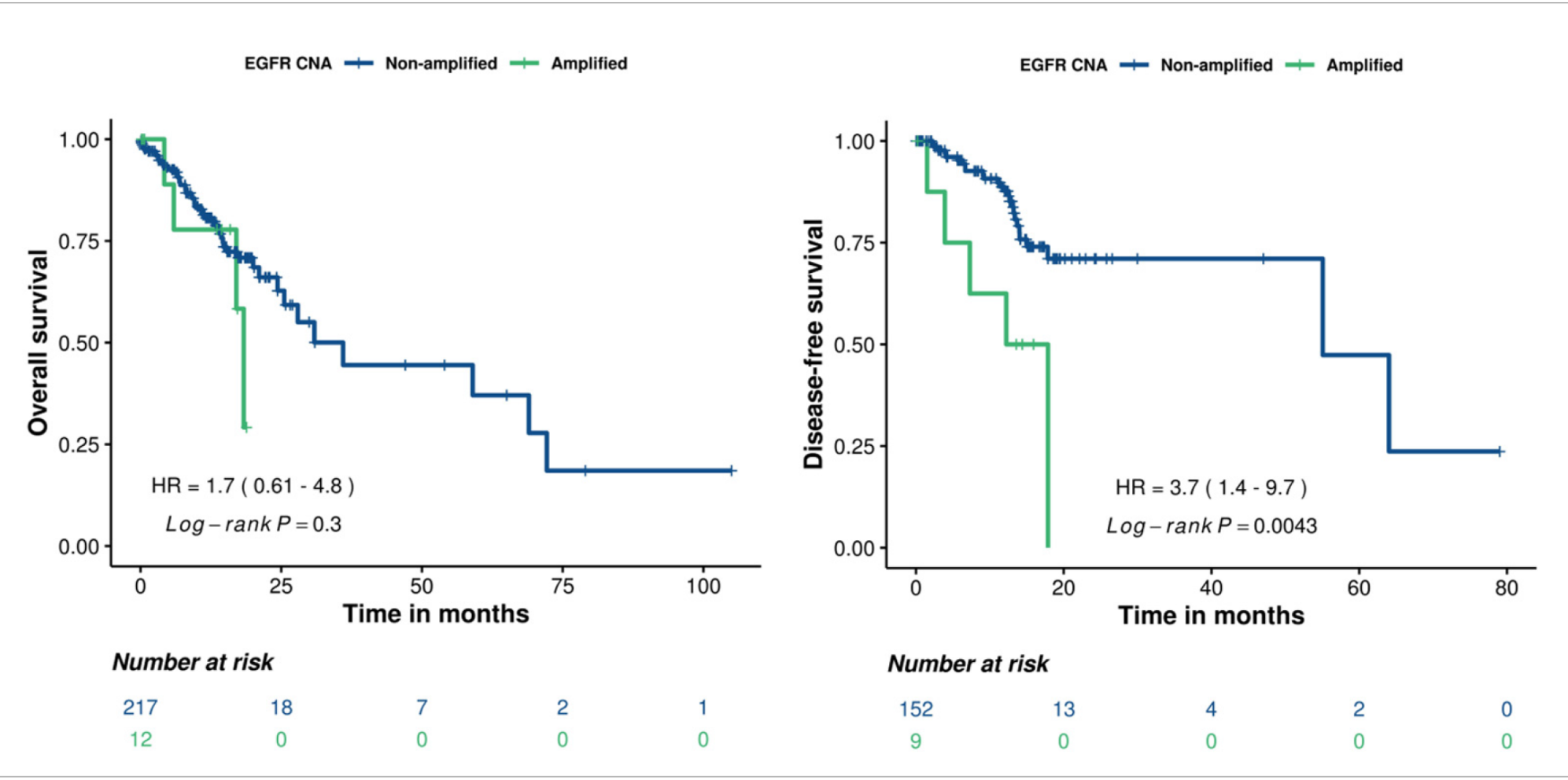



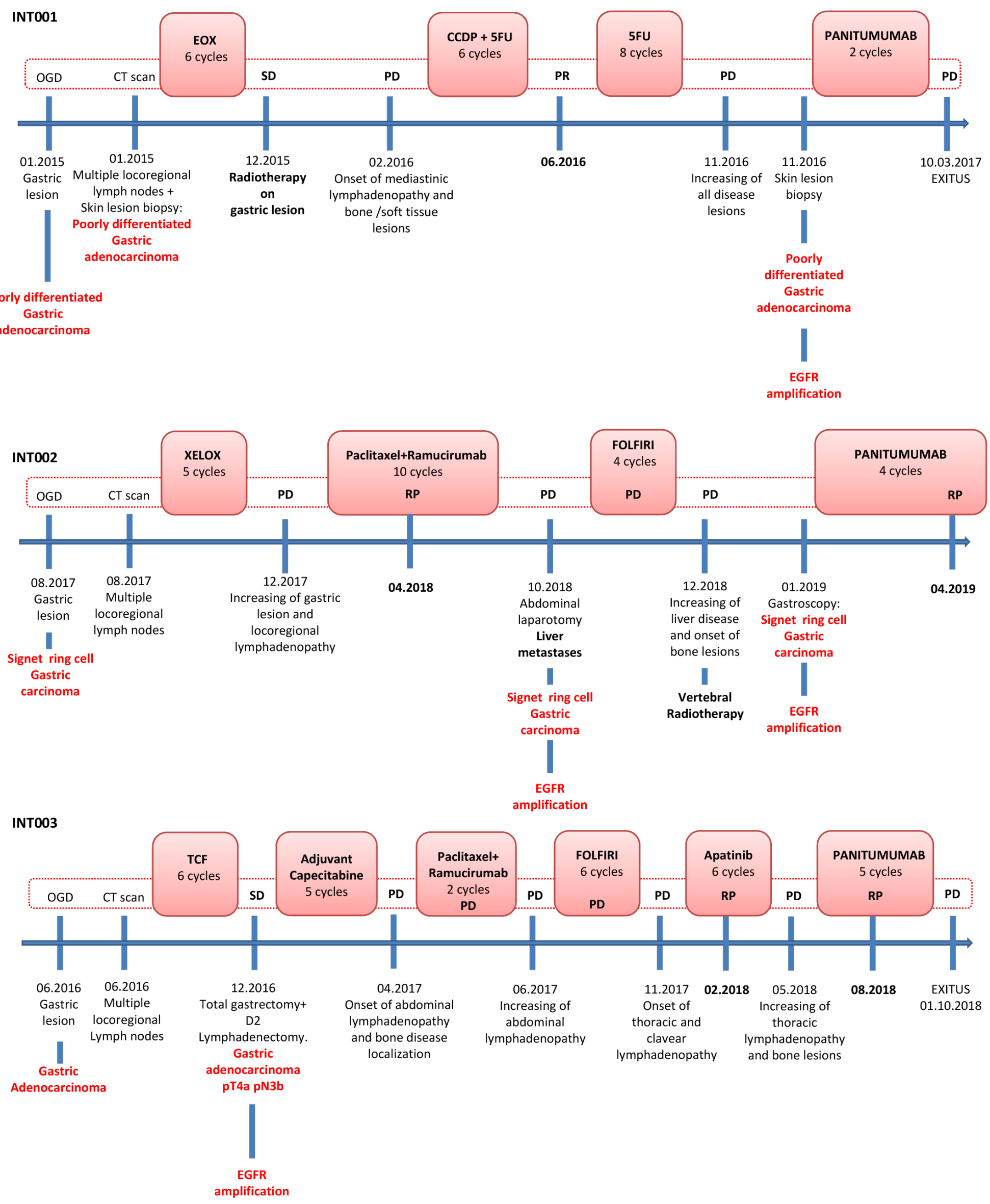
A

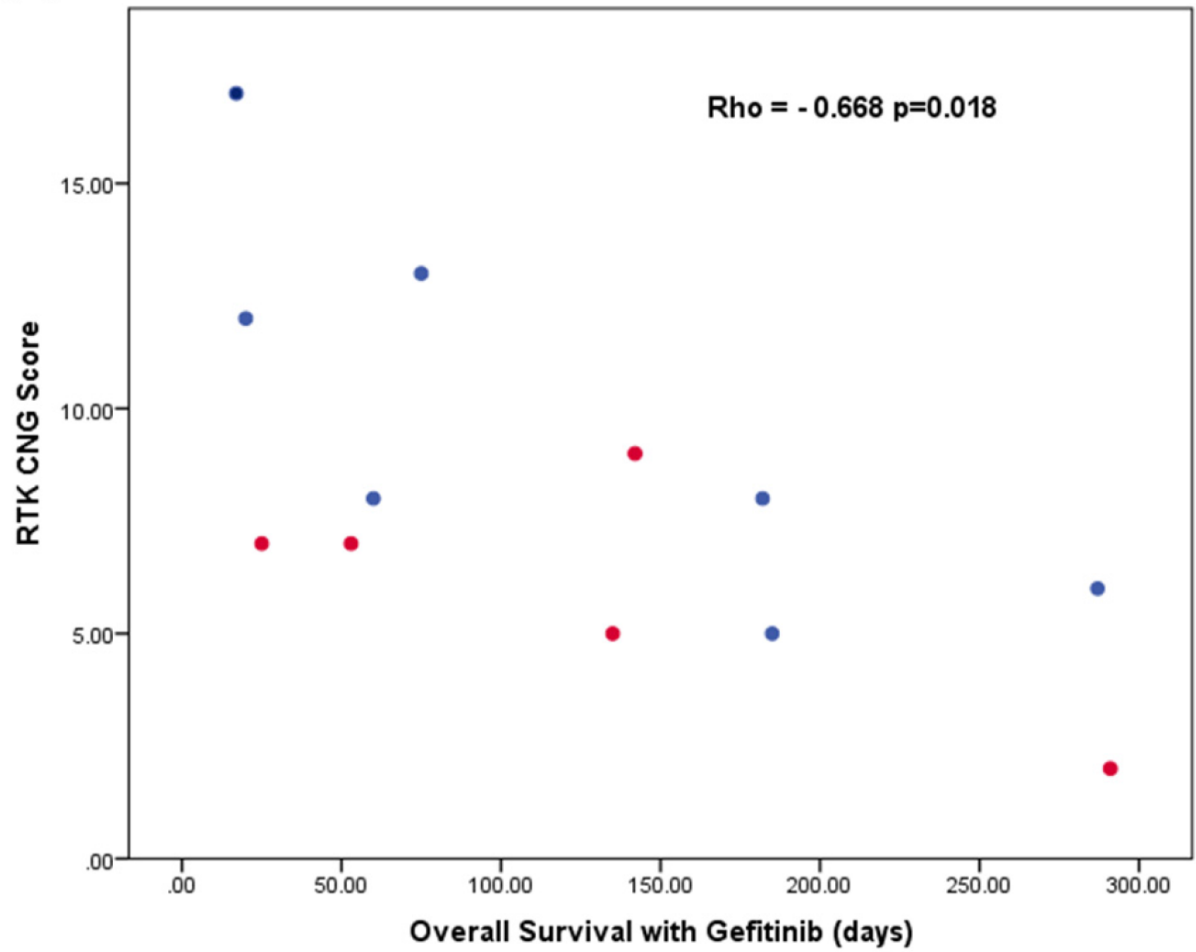

high polisomy EGFR amplification.

B

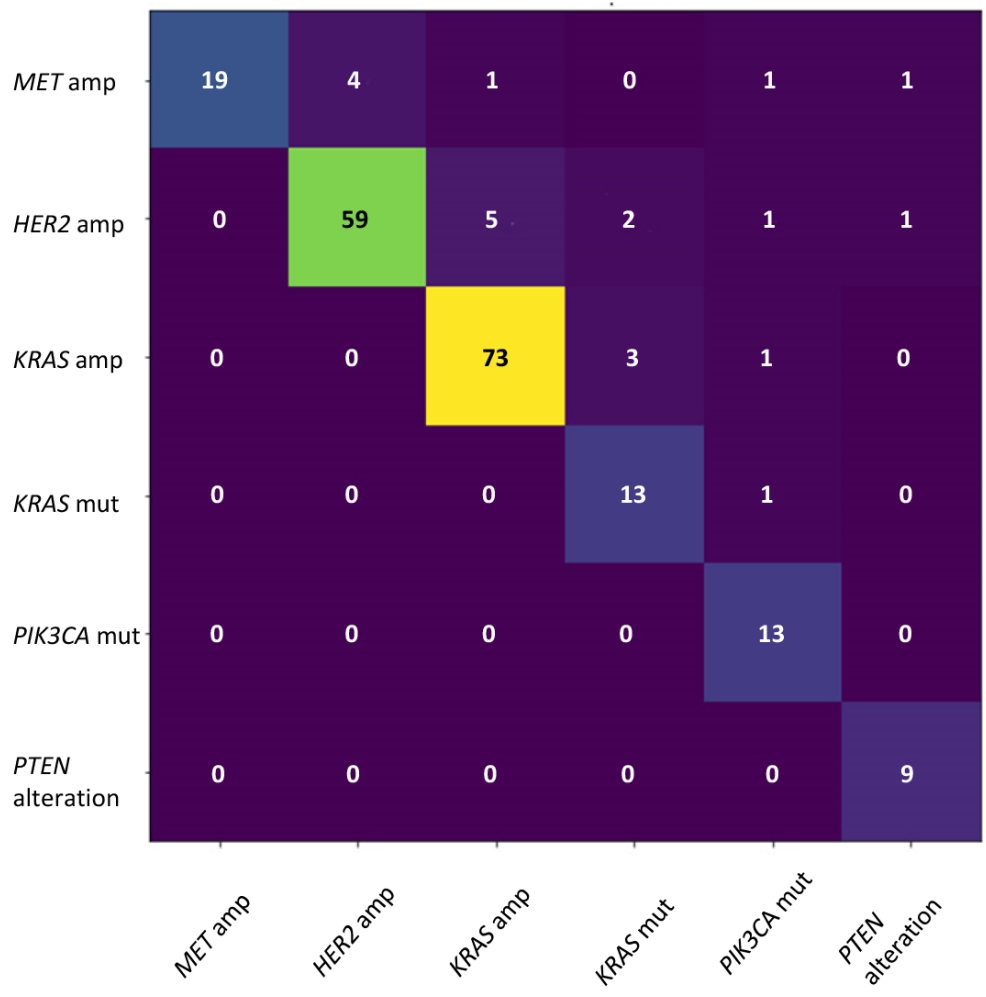
Research. 


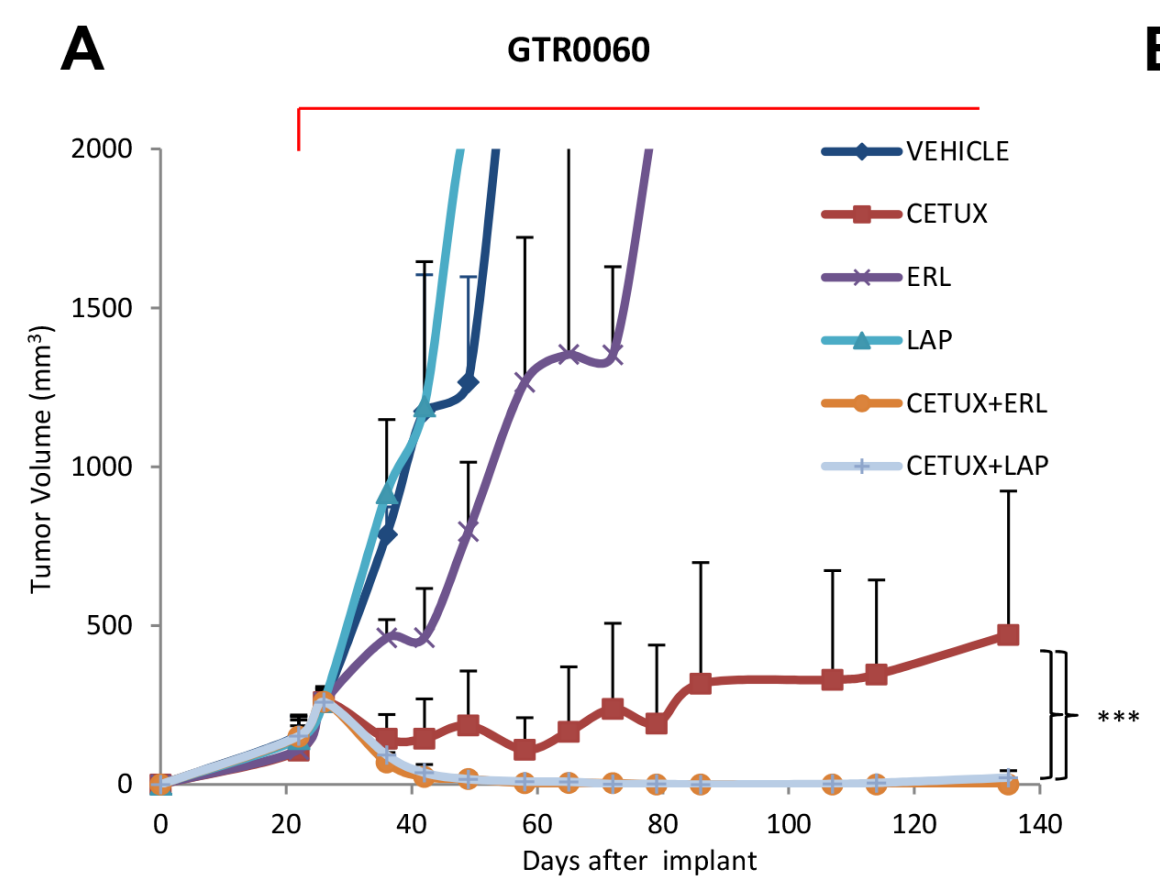

B

Figure 5
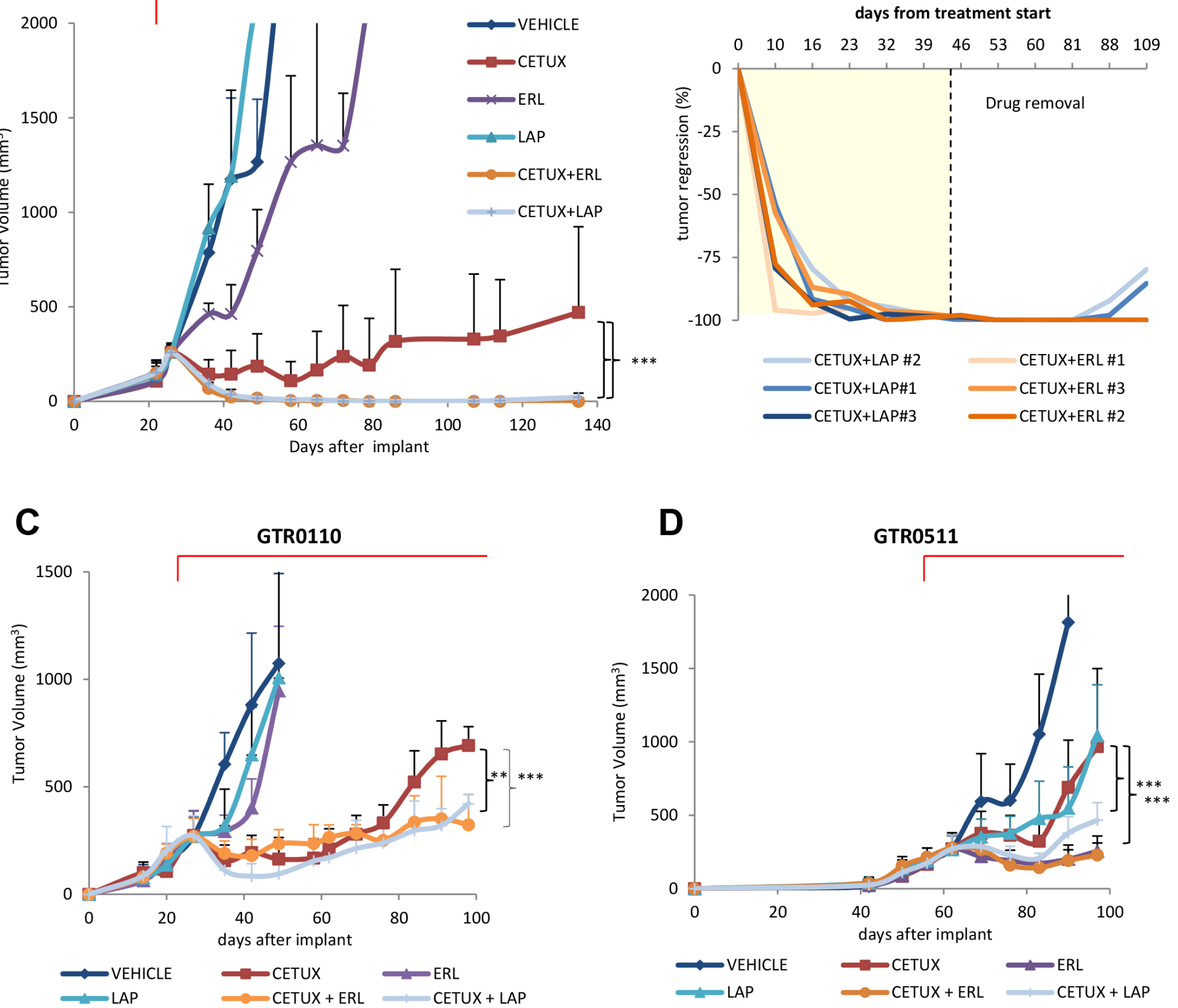

E
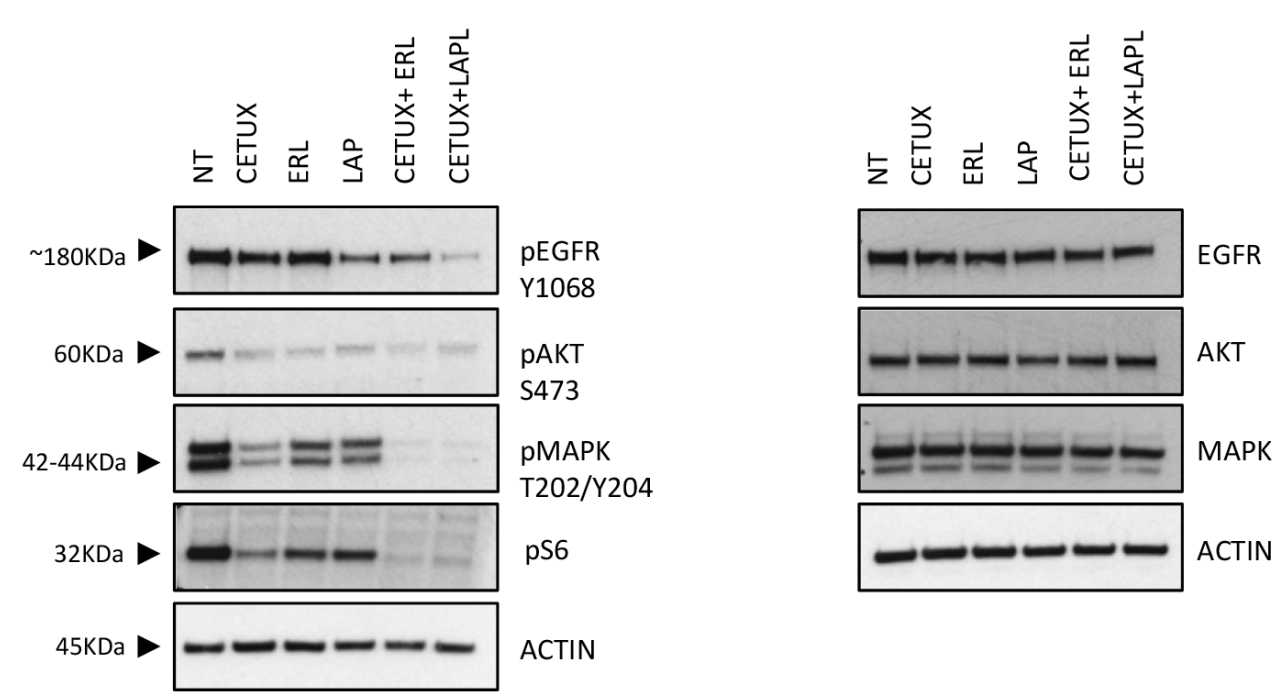

Downloaded from clincancerres.aacrjournals.org on February 25, 2021. ( 2021 American Association for Cancer Research. 
A
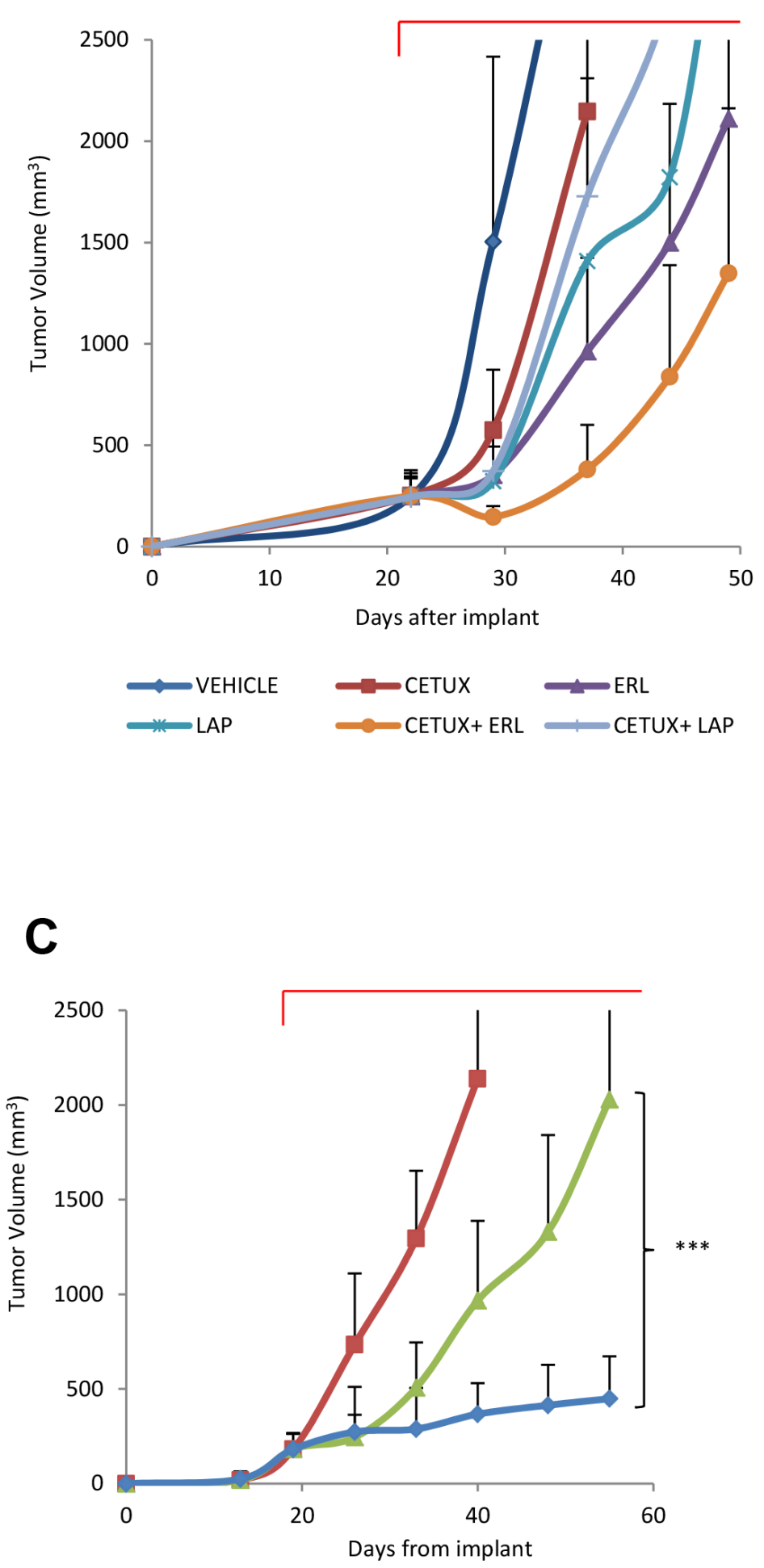

$\longrightarrow$ VEHICLE $\longrightarrow$ ERL $\leadsto$ ERL+EVEROL
B

\begin{tabular}{|c|c|c|c|c|c|}
\hline $\begin{array}{c}\text { Hugo } \\
\text { Symbol }\end{array}$ & $\begin{array}{c}\text { Protein } \\
\text { Change }\end{array}$ & cDNA Change & $\begin{array}{c}\text { Variant } \\
\text { Classification }\end{array}$ & dbsnp Site & Allele Fraction \\
\hline TSC2 & p.M1300V & c.3898A>G & Missense & NOVEL & $\mathbf{0 , 4 6 3 9 1 8}$ \\
\hline TSC2 & p.R1438Q & c.4313G>A & Missense & COSMIC & $\mathbf{0 , 5 3 9 8 2 3}$ \\
\hline
\end{tabular}

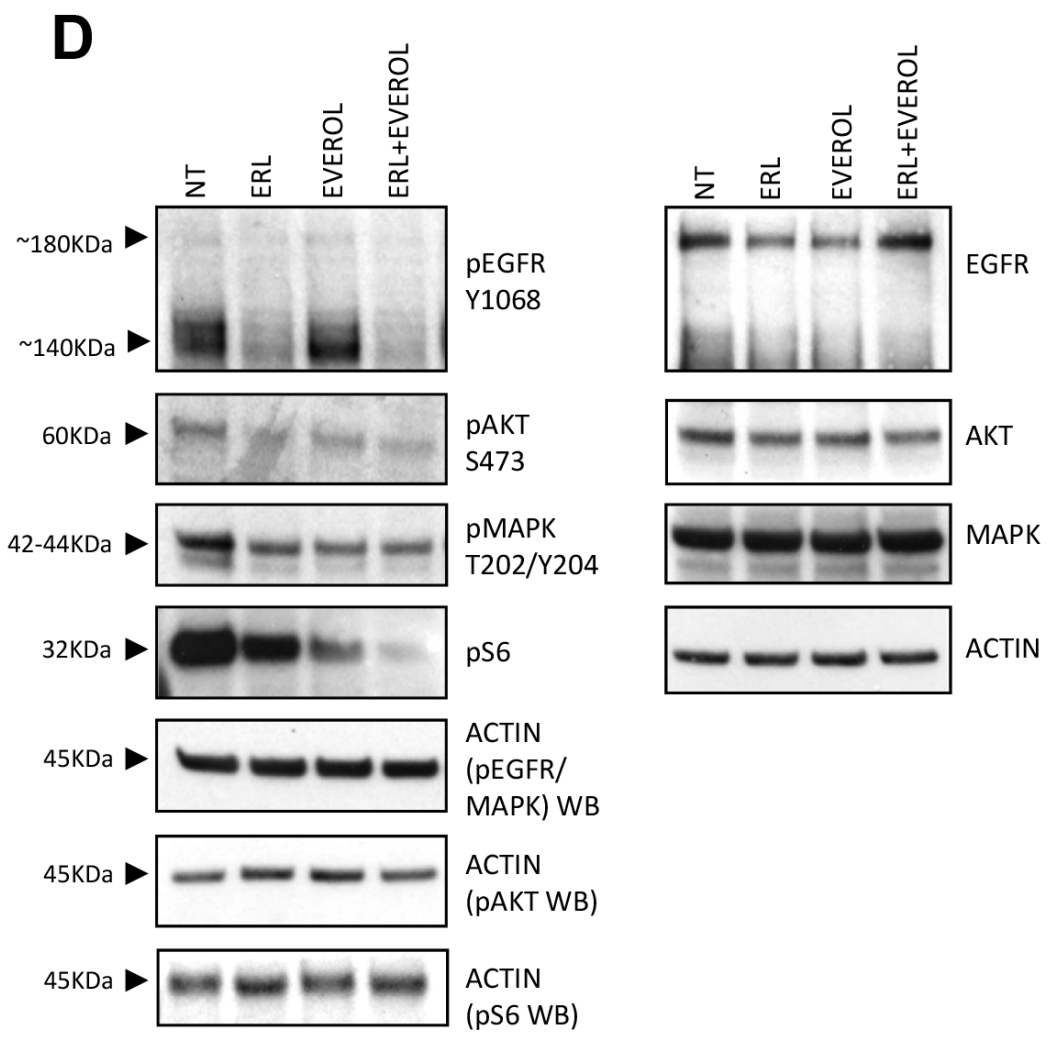




\section{Clinical Cancer Research}

\section{Optimized EGFR blockade strategies in EGFR addicted gastroesophageal adenocarcinomas}

Simona Corso, Filippo Pietrantonio, Maria Apicella, et al.

Clin Cancer Res Published OnlineFirst February 4, 2021.

\section{Updated version Access the most recent version of this article at:} doi:10.1158/1078-0432.CCR-20-0121

Supplementary Access the most recent supplemental material at:

Material http://clincancerres.aacrjournals.org/content/suppl/2021/02/04/1078-0432.CCR-20-0121.DC1

Author Author manuscripts have been peer reviewed and accepted for publication but have not yet Manuscript been edited.

E-mail alerts Sign up to receive free email-alerts related to this article or journal.

Reprints and To order reprints of this article or to subscribe to the journal, contact the AACR Publications Subscriptions Department at pubs@aacr.org.

Permissions To request permission to re-use all or part of this article, use this link http://clincancerres.aacrjournals.org/content/early/2021/02/03/1078-0432.CCR-20-0121. Click on "Request Permissions" which will take you to the Copyright Clearance Center's (CCC) Rightslink site. 